\title{
The dominating mechanisms for the formation of solute-rich clusters in steels under irradiation.
}

N. Castin ${ }^{(1, *)}$, G. Bonny ${ }^{(1)}$, A. Bakaev ${ }^{(1)}$, F. Bergner ${ }^{(2)}$, C. Domain ${ }^{(3)}$, J.M. Hyde ${ }^{(4,5)}$, L. Messina $^{(6)}$, B. Radiguet ${ }^{(7)}$ and L. Malerba ${ }^{\left(1,8,{ }^{*}\right)}$

(1) Studiecentrum voor Kernenergie - Centre d'Études de l'énergie Nucléaire (SCK•CEN), NMS Unit, Boeretang 200, B2400 Mol, Belgium.

(2) Helmholtz-Zentrum Dresden-Rossendorf, Institute of Resource Ecology, P.O. Box 510119, 01314 Dresden, Germany.

(3) EDF-R\&D, Département Matériaux et Mécanique des Composants (MMC), Les Renardières, F-77818 Moret sur Loing Cedex, France.

${ }^{(4)}$ National Nuclear Laboratory, Culham Science Centre, Abingdon, Oxfordshire OX14 3DB, UK.

${ }^{(5)}$ Department of Materials, University of Oxford, Parks Road, Oxford OX1 3PH, UK

${ }^{(6)}$ KTH Royal Institute of Technology, Nuclear Engineering, 10691 Stockholm, Sweden.

(7) Groupe de Physique des Matériaux, Université et INSA de Rouen, UMR CNRS 6634, B.P.

12, 76801 Saint-Etienne du Rouvray Cedex, France.

(8) Centro de Investigaciones Energéticas, Medioambientales y Tecnológicas (CIEMAT), Avda. Complutense 40, 28040 Madrid, Spain.

${ }^{(*)}$ Corresponding author: nicolas.m.b.castin@gmail.com

\section{Abstract}

The formation of nano-sized, coherent, solute-rich clusters (NSRC) is known to be an important factor degrading the macroscopic properties of steels under irradiation. The mechanisms driving their formation are still debated. This work focuses on low-Cu reactor pressure vessel (RPV) steels, where solute species are generally not expected to precipitate. We rationalize the processes taking place at the nanometre scale under irradiation, relying on the latest theoretical and experimental evidence on atomic-level diffusion and transport processes. These are compiled in a new model, based on the object kinetic Monte Carlo (OKMC) technique. We evaluate the relevance of the underlying physical assumptions by applying the model to a large variety of irradiation experiments. Our model predictions are compared with new experimental data obtained with atom probe tomography and small angle neutron scattering, complemented with information from the literature. The results of this study reveal that the role of immobilized selfinterstitial atoms (SIA) loops dominates the nucleation process of NSRC. 


\section{Introduction}

Assessing and predicting embrittlement of reactor pressure vessel (RPV) steels as a function of the neutron dose is of crucial importance for the safe operation of nuclear power plants. Substantial effort has been put over the last decades not only to develop socalled dose-damage correlations of semi-empirical nature [1], but also to ensure they are based on mechanistic understanding [2]. The data to which these correlations are fit come from vessel surveillance [3], decommissioned vessels [4], or irradiation campaigns in materials testing reactors (MTR) [5-7]. For a longer-term application, effort has been put to develop fully physically informed suites of computer simulation codes, aimed at predicting RPV steel radiation hardening [8,9], including microstructural examination studies in support of modelling [10-28]. These studies revealed that radiation hardening in RPV steels, as well as in other types of iron alloys such as high-Cr ferritic-martensitic (FM) materials, is mainly the consequence of the formation of high densities $\left(\sim 10^{23} \mathrm{~m}^{-3}\right)$ of nanometre-size solute-rich clusters (NSRC), which act as obstacles to dislocation motion $[29,30]$. Importantly, NSRC are also found in steels containing low quantities of $\mathrm{Cu}$ for which all solute species are found below their solubility limit. For this reason, this work focuses on low $\mathrm{Cu}$ steels.

Embrittlement in RPV steels ensues mainly from hardening, due to the fact that the obstruction of dislocation motion hinders the blunting of crack tips. Contributions from embrittlement without hardening cannot be excluded either, due to solute segregation at grain boundaries that can result in intergranular fracture [31], but these are generally expected to have a minor role in determining brittle behaviour.

Microstructure examination experiments show that, after irradiation, the NSRC contain varying concentrations of $\mathrm{Cu}, \mathrm{Mn}, \mathrm{Ni}, \mathrm{Si}$ and $\mathrm{P}$, as well as $\mathrm{Cr}$ in the case of F-M steels. Due to their small size and coherence with the matrix (body centred cubic - bcc - structure), they are not easily resolvable to conventional transmission electron microscopy (TEM). Their existence has been revealed mainly through other microstructural characterization techniques, such as atom probe tomography (APT) [10-21,32] and small angle neutron scattering (SANS) [24-28]. Recently, though, modern scanning TEM (STEM) techniques coupled with energy dispersive spectroscopy (EDS) allowed these clusters to be resolved as well [33].

Empirical correlations reveal a direct proportionality between hardening $\left(\Delta \sigma_{y}\right.$, where $\sigma_{\mathrm{y}}$ is the yield strength and $\Delta$ indicates the increase) or embrittlement $\left(\Delta \mathrm{T}_{\mathrm{NDT}}\right.$, where $\mathrm{T}_{\mathrm{NDT}}$ is the nil ductility temperature), and the square root of the volume fraction of these clusters $[28,34]$. Physical models show the dependence of hardening on the square root of density times size $[35,36]$. Therefore, a quantitative assessment of radiation-induced hardening and embrittlement as a function of radiation dose and dose-rate requires a correct description of the kinetics of the atomic-level processes that lead to the formation of the NSRC observed by APT, SANS, STEM-EDS, etc. The mentioned experimental techniques provide invaluable information concerning number density, size and composition of NSRC after irradiation experiments. However, only physical models can describe the atomiclevel processes that lead to their formation in sufficient detail, allowing the correct assessment of these quantities also without the need of microstructural examinations that are not routinely performed on materials in operation, for any dose and at any dose-rate. In particular, the knowledge of the kinetics of formation is essential with a view to predicting correctly the build-up of damage versus dose, so as to be able to anticipate, as much as possible, the materials degradation up to doses of relevance for, e.g., long term operation. 
The involved processes stem from irradiation, which introduces in crystalline materials an excess of point defects (vacancies and self-interstitial atoms) that are produced in cascades of atomic displacements (displacement cascades). These are complex, collective processes that have been studied for decades by means of atomistic simulation tools, and are triggered by the collision of the incoming energetic particles (neutrons in nuclear reactors) with the atoms of the lattice [37-39].

Because of the complexity of the physical processes induced by the continuous injection of point defects during irradiation, the mechanisms leading to the subsequent formation of NSRC are still debated. Previous works emphasized the role of driving forces from thermodynamics [40-45]. In this framework the role of radiation defects (particularly vacancies) would only be to accelerate the diffusion processes that lead the system to thermodynamic equilibrium in terms of phase separation, because their concentration is well-above the equilibrium one. In this view, NSRC formation in RPV steels would essentially be an irradiation-enhanced process. In contrast, other researchers have suggested that the evolution of the system is dominantly driven by mechanisms specific to irradiation [16, 21, 46, 47], i.e. considering as crucial the role of self-interstitial atoms (SIA). In this perspective, NSRC formation in RPV steels would be largely a radiationinduced process. The model proposed in Ref. [43] already suggested that both mechanisms must be acting, highlighting the role of "cascade-induced nucleation" (this is the expression used in [43]). This work aims at providing a quantitative estimate of the dominant mechanisms that govern the formation of NSRC, considering both the role of vacancies and SIAs, in the light of the latest theoretical and experimental evidence concerning atomic-level processes of diffusion and transport. For this reason, i.e. to collect and juxtapose all the relevant information, section 2 is devoted to reviewing the work that has been done recently on these subjects. New experimental evidence from APT and SANS obtained in this work is reported, in complement to information from the literature. This review is instrumental to be able to draw a detailed and complete map of the mechanisms that can be considered responsible for the nucleation and growth of the NSRC. Next, in section 3, these mechanisms are incorporated in a new model that describes the evolution of the radiation-induced nano-features in ferritic steels, by implementing for the first time explicit solute transport in an object kinetic Monte Carlo framework. The formation of NSRC is one of the natural outputs of this model, which encompasses all features related with irradiation effects at the nanometre scale, importantly including point-defects clusters. The model is then applied, in section 4, to simulate irradiation experiments on a wide variety of RPV steels and model alloys. Direct comparison between the model prediction and experimental evidence with APT enables the relevance of the physical assumptions that underlie the model to be tested. Our findings are then discussed in section 5 , where we deduce what is the dominant mechanism at play.

\section{Mechanism driving the formation of nano-sized solute rich clusters}

\subsection{Theoretical considerations}

In this section we explain in detail our model, showing that it is rooted in extensive theoretical studies performed over the last decade. Calculations with density functional theory (DFT) reveal that all the solute atoms that are typically observed in NSRC $(\mathrm{Cu}, \mathrm{Mn}$, 
$\mathrm{Ni}, \mathrm{Si}$ and P) exhibit attractive binding energies with vacancies [48-50]. This has two consequences:

1. These solutes are transported by single vacancies via a drag mechanism. To be more precise, the flux of vacancies is coupled to the flux of these solutes and oriented in the same direction (positive correlation). This naturally leads to segregation of these atoms at sinks where vacancies are absorbed. Messina et al. [49] demonstrated that, contrary to widespread perception, this dragging is a general process, common to many solute species in Fe. The dragging is due to the fact that the presence of the solute modifies locally the energy barriers for vacancy migration. The model by Messina et al. was based on an exact analytical self-consistent mean-field approach, making use of DFT-calculated migration barriers. The conclusion was that, in the infinite dilution limit, the vacancy-solute flux stemming from this positive correlation is sufficiently strong for $\mathrm{Cu}, \mathrm{Ni}, \mathrm{Mn}, \mathrm{Si}$ and $\mathrm{P}$ to produce radiation-induced segregation at point defect sinks up to relatively high temperatures. The range includes $300^{\circ} \mathrm{C}$, which is the approximate operation temperature of the RPV, and in fact extends to the whole ferromagnetic temperature range. On the contrary, a negative correlation and thus an absence of drag was predicted for typical solutes that are present in RPV and also F/M steels, but are never found in NSRC, e.g. Mo.

2. Stable vacancy-solute clusters are expected to form. In this work (see later in section 3), we have evaluated the magnitude of the collective binding energies between solutes and vacancies by performing a large amount of DFT calculations, up to triplet configurations (see supplementary material for the full tabulation). The results of these calculations demonstrate that solute-vacancy pairs can remain stable defects at RPV-relevant temperatures (near $300^{\circ} \mathrm{C}$ ). We thus expect these configurations to be nuclei for the formation of larger clusters.

DFT also reveals a significant interaction between solute atoms and SIA defects, namely:

1. Some solutes, specifically $\mathrm{P}, \mathrm{Mn}$ and $\mathrm{Cr}$, are transported by SIAs, via the formation and migration of mixed dumbbells [50]. By definition, this implies the migration of the involved point-defects and solutes in the same direction. Hence, once again, transport of solutes towards point-defect sinks occurs. It has been calculated that these three solutes are indeed prone to segregation at point defect sinks, due to SIA transport, up to high temperatures [48].

2. It has also been shown that, in addition to small SIA clusters [51], small prismatic loops of self-interstitial nature that may form directly in displacement cascades interact strongly and attractively with solutes, especially with $\mathrm{P}, \mathrm{Si}, \mathrm{Mn}, \mathrm{Cu}$ and $\mathrm{Ni}$ (in order of strength) [52]. The interaction energy depends on whether the solute interacts with the centre or the edge of the loop; it ranges between 0.2 and $0.5 \mathrm{eV}$ and can be as high as $1 \mathrm{eV}$ in the case of P. In addition, both experiments and atomistic studies have shown that carbon-vacancy complexes (possibly nitrogen-vacancy and oxygen-vacancy complexes as well [53]) form abundantly under irradiation [54,55] and act as very efficient traps for gliding prismatic loops [56], with interaction energies as high as $1.3 \mathrm{eV}$ [57]. The existence of an affinity between solute atoms and loops, as revealed by DFT, has been extended to atomistic studies with interatomic potentials using Metropolis Monte Carlo techniques. These studies revealed that $\mathrm{Cu}$, $\mathrm{Mn}, \mathrm{Ni}$, and $\mathrm{P}$ ( $\mathrm{Si}$ has not been studied because of the absence of a suitable $\mathrm{Fe}-\mathrm{Si}$ interatomic potential) naturally tend to accumulate around extended defects, such as dislocation loops and lines. They thus create energetically stable clouds around them, as well as precipitates (the simulations specifically suggest the formation of $\mathrm{Cu}$ and 
NiMn B2 phase precipitates, or Mn-rich regions, attached to dislocation loops and lines) [58,59].
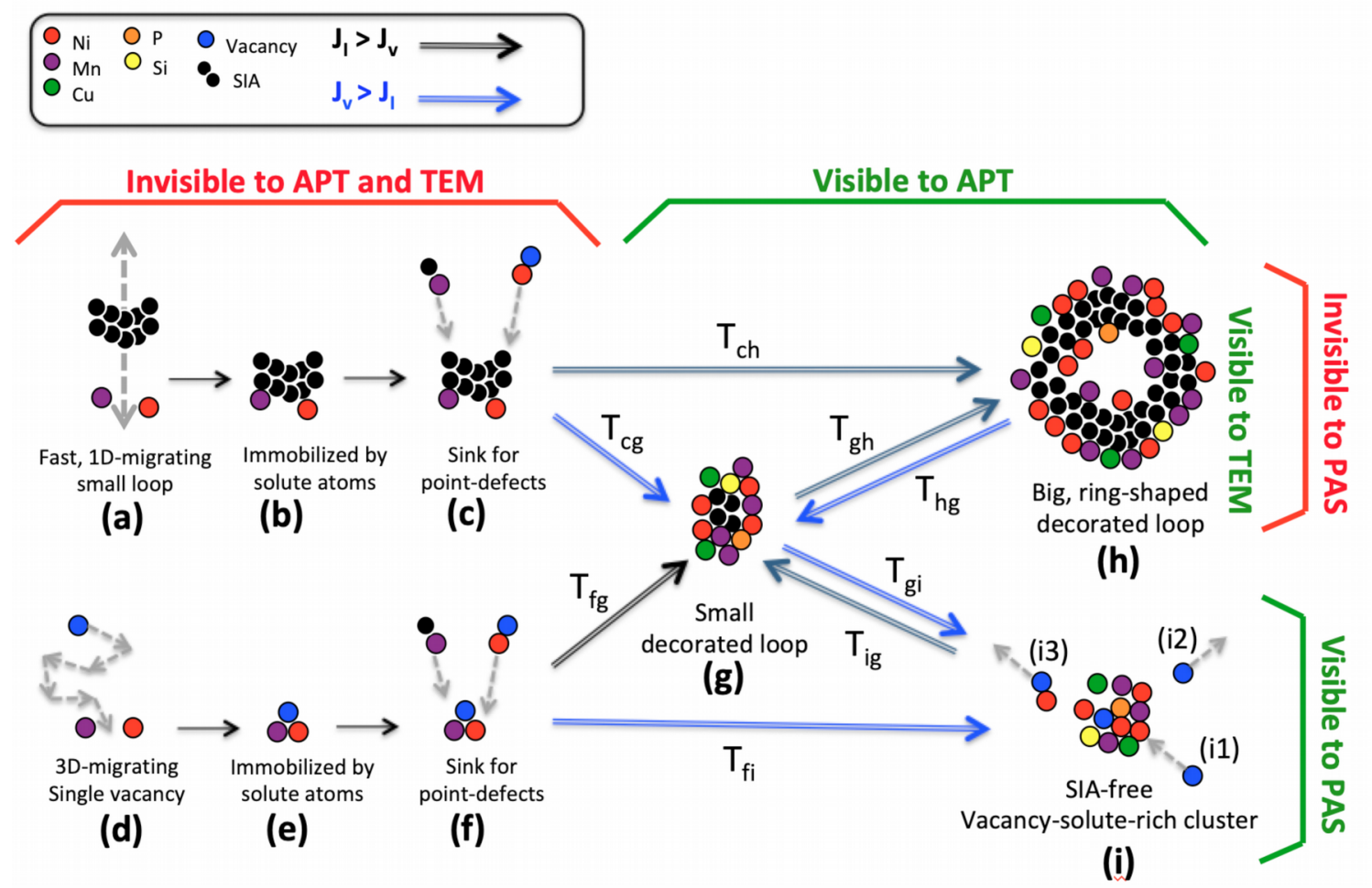

Fig. 1 - Hypothetical scenario for the formation of NSRC. Solute atoms and point-defects are represented by coloured circles (see legend). Fe is the matrix, not depicted in the figure. $\mathrm{J}_{\mathrm{I}}$ and $\mathrm{J}_{\mathrm{V}}$ denote the incoming flux in interstitial defect or vacancy defects, respectively.

Based on these considerations, we propose here the general scenario illustrated in Fig. 1 for the nucleation of NSRC in steels under irradiation. A first sketch of this possible scenario has been put forward for FeMnNi alloys [60-61], but it is here generalised and fully developed for the first time. To start with, two distinct mechanisms of NSRC nucleation may exist:

1) In Fig. 1a-1c, nucleation occurs because of the interaction between SIA-defects and solute atoms.

a. In Fig. 1a, small, glissile, one-dimensionally migrating SIA clusters (small prismatic dislocation loops) are created in the material during or after displacement cascades $[37-39,62]$. Their migration energy is $\sim 0.1 \mathrm{eV}$ or less in the absence of interaction with impurities [63].

b. In Fig. 1b, the migrating SIA cluster encounters solute atoms (or carbon-vacancy complexes) along its glide prism. Its migration is hence temporarily stopped, due to the attractive interaction, the strength of which depends on the nature of the solute (or complex) and its relative position with respect to the loop (centre or edge) [52]. While in most cases a relatively weak binding energy is involved, some solutes, like $\mathrm{P}$, or pairs of solutes, as well as carbon-vacancy complexes, can trap the loop with energy values around, or in excess of, $1 \mathrm{eV}$. At the RPV operation temperature, this energy is sufficient to immobilise the defect completely. These types of encounters are more likely with increasing solute concentration. DFT 
studies [52] show, in addition, that the interaction with multiple solutes adds up in strength, thereby making weakly interacting solutes like $\mathrm{Cu}, \mathrm{Ni}$ and $\mathrm{Mn}$ also able to act as traps, when combined. Since a single prismatic dislocation loop cannot, by gliding, drag substitutional solutes with it, this interaction does not lead to net solute transport [64].

c. I n Fig. 1c, provided that the time of immobilization is long enough, the loop effectively becomes a sink for migrating point defects. Since many point-defects are expected to carry solute atoms, there is a high chance that new solutes are thereby deposited on the loop. The loop remains thus trapped with increasing strength, eventually losing any possibility of migrating away from the trapping solutes, while the local concentration of solutes keeps increasing. The latter effect, combined with the strong affinity that some solutes have for loops [52], makes these locations ideal for the precipitation of equilibrium thermodynamic phases, including some that would not precipitate if the concentration remained equal to the nominal one [65]. However, this process favours the accumulation of solutes irrespective of whether they do or do not form a known stable thermodynamic phase. Thus, the composition of NSRCs may, but need not, correspond to the composition of stable thermodynamic phases. In addition, a recent thermodynamic study [66] based on DFT revealed that the number of phases that may exist in RPVsteel like systems is extremely large, well beyond the number of phases included e.g. in Thermo-Calc [67]. Thus, the variety of potentially possible compositions is really wide.

2) In Fig. 1d-1f, a similar scheme to that above is anticipated, this time pivoting around the interaction between vacancy defects and solute atoms:

a. Like SIA defects, migrating vacancies, or migrating small vacancy-clusters in Fig. 1d, are likely to encounter solute atoms. Mutual binding between vacancies and solutes leads to the formation of complexes, as revealed by our DFT study (see supplementary material). Small solute-vacancy clusters as depicted in Fig. 1e can therefore form.

b. Consequently, in Fig. 1f, these vacancy-solute clusters are also expected to play the role of sinks for other migrating point-defects. As the latter carry solute atoms, the local enrichment increases (similarly to Fig. 1c). It is important to note that, in contrast to SIA-solute clusters in Fig. 1c, solute-vacancy clusters can dissolve at RPV-relevant temperatures. Since the magnitude of binding energy for a single vacancy, or a vacancy-solute pair is of the order of 0.3-0.7 eV (see supplementary material), the emission of single defects from the cluster constantly occurs, also provoking the dissolution of solute species.

Following the nucleation events described above, Fig. 1 also depicts possible growth mechanisms, via transitions indicated by thick arrows. Since loops cannot transport solutes, and assuming that the transport of solutes clusters by vacancies is negligible, no direct coalescence of NSRC is expected. Instead, immobilized defects in Fig. 1c and Fig $1 \mathrm{f}$ are affected by the incoming flux of mobile single point defects, which is always associated with a positive flux of solute atoms. Given a particular defect, we define as $\mathrm{J}_{\mathrm{I}}$ the incoming flux of self-interstitial atoms towards it, and as $\mathrm{J}_{\mathrm{v}}$ the incoming flux of vacancies towards it:

c. The case $\mathrm{J}_{\mathrm{I}}>\mathrm{J}_{\mathrm{v}}$, i.e. incoming flux predominantly of SIA kind (including single SIAs but also small SIA clusters not yet immobilized by solute atoms), thus outnumbering the incoming flux of vacancies, is depicted by thick black arrows in Fig. 1. A vacancy-solute cluster in Fig. 1f would thus follow the transition $\mathrm{T}_{\mathrm{fg}}$, gradually losing its vacancy content, until becoming a small decorated loop like in 
Fig. 1g. Should the magnitude of $\mathrm{J}_{\mathrm{I}}$ be large enough, the defect can even follow the $\mathrm{T}_{\mathrm{gh}}$ transition and become a large loop like in Fig. (1h). Since the binding between solutes and loops is stronger at the periphery of the latter [52], solutes are expected to accumulate there, giving rise to the ring shape depicted in the figure. Defects originally nucleated as in the Fig. 1a-1c events also follow the same fate.

d. The case $\mathrm{J}_{\mathrm{v}}>\mathrm{J}_{\mathrm{l}}$, i.e. the net balance of incoming point defects is higher for vacancies than SIA, is depicted by thick blue arrows in Fig. 1. Small loops like in Fig. 1g are in this case expected to shrink, eventually becoming SIA-free defects, as depicted in Fig. 1i. Defects originally nucleated in Fig. 1d-1f events also evolve towards this configuration.

Our model in Fig. 1 therefore foresees the existence of three distinct kinds of solute-rich clusters. They are all visible using APT, provided that the total number of solute atoms involved is large enough.

- In Fig 1g, small loops are not visible to the TEM because the number of SIA defects is too small. They are, however, visible to the APT thanks to their solute decoration, which would be seen as a spherical cloud of solute atoms, while the SIA content cannot be resolved by this experimental technique. It is important to note that the interstitial nature of the loop makes it very unlikely that a SIA is released from it, because the binding energy of SIAs to mother clusters is on the order of several eV, except for clusters of 2-3 SIA [68]. On the other hand, vacancies approaching the defect will recombine and therefore will not produce any solute transport or rearrangement. Consequently, it is very unlikely that solutes can be removed from a decorated selfinterstitial loop. They can thus only grow in number of solute atoms.

- In Fig. 1h, big solute-decorated loops are foreseen as a possible limiting case from Fig $1 \mathrm{~g}$, given favourable conditions. These defects are visible to the TEM, because of their loop nature. Their typical ring-shape is likely to be visible to the APT as well, if homogeneously decorated by solute atoms. However, solute atoms may also accumulate inhomogeneously, giving rise to groups of separate solute clusters lying on the habit plane of the loop.

- In Fig. 1i, the solute-rich cluster is free of SIA. It has a vacancy content instead, which can be single vacancies, or small vacancy clusters. It is thus not visible to the TEM, but may be seen as a diffuse cloud of atoms by APT; moreover, positron annihilation spectroscopy (PAS) will be sensitive to the presence of these clusters and may help detect them. The solute cluster may grow further (positive incoming flux of solute atoms), but may also dissolve, especially if not corresponding to any stable phase. Indeed, as illustrated in Fig. 1i, here the release of vacancies (Fig. 1i2) and thus also the emission of solute-vacancy pairs (Fig. 1i3), is possible, so these types of clusters are thermally less stable. This reminds of the so-called "unstable matrix damage" sometimes invoked to explain neutron dose rate effects in RPV steel embrittlement $[69,70]$.

In summary, the conceptual model depicted in Fig. 1 and based on the most relevant theoretical evidence anticipates that NSRC nucleation can take place from the mutual interaction between solute atoms with vacancies and SIA defects. Because of the strong affinities between point-defects and solute atoms, 'nuclei' for NSRC can be as small as one or two solutes. The solute-solute interaction and relevant thermodynamic driving forces will only have a role (if any) at a later stage, when the local concentration has grown sufficiently to trigger thermodynamic processes. In other words, the catalysing effect of point defect clusters requires no solute-cluster critical size that should be overcome so that the interface energy is compensated by the gain in free energy due to phase 
separation, as in a "classical" nucleation-and-growth scenario driven by single vacancy diffusion and thermodynamic forces. Nor must the compositions of the NSRC correspond necessarily to thermodynamic phases, strongly or weakly stable, out of all those that are possible [66]: non-equilibrium compositions may also appear and be stable, because of the affinity with point-defects and their clusters [58].

\subsection{Experimental evidence}

Many pieces of experimental evidence support our conceptual model for NSRC formation, as depicted in Fig. 1.

To start with, APT frequently reveals segregation of solute atoms on various crystal defects such as dislocation lines, grain boundaries or carbide matrix interfaces in irradiated RPV steels [16-23, 32]. This clearly supports the hypothesis that solute atoms are continuously dragged by single point defects towards point-defect sinks, irrespective of the magnitudes of solute-solute interactions.

Next, a large number density $\left(\sim 10^{22} \mathrm{~m}^{-3}\right.$ to $\left.10^{2}{ }^{3} \mathrm{~m}^{-3}\right)$ of roughly spherical NSRC is systematically reported in nearly all APT studies on irradiated steels [10-24]. A typical image obtained from APT is shown in Fig. 2. These are clearly associated with the defects depicted in Fig. $1 \mathrm{~g}$ and Fig 1i, as discussed above. A collection of experimental data, many of which part of this work, is summarized in Tab. 1, for a total of 10 materials, that are divided in three categories: (1) Model alloys for RPV steels; (2) base metals in RPV steels; (3) weld metals in RPV steels. Irradiation conditions in terms of dose rate and temperatures also differ through Tab. 1, as sample materials were irradiated either in operating nuclear power plants, or in MTR facilities. The majority of these data were obtained during the LongLife European project [71], but had remained so far unpublished (they are therefore denoted as "this work" in Tab. 1).

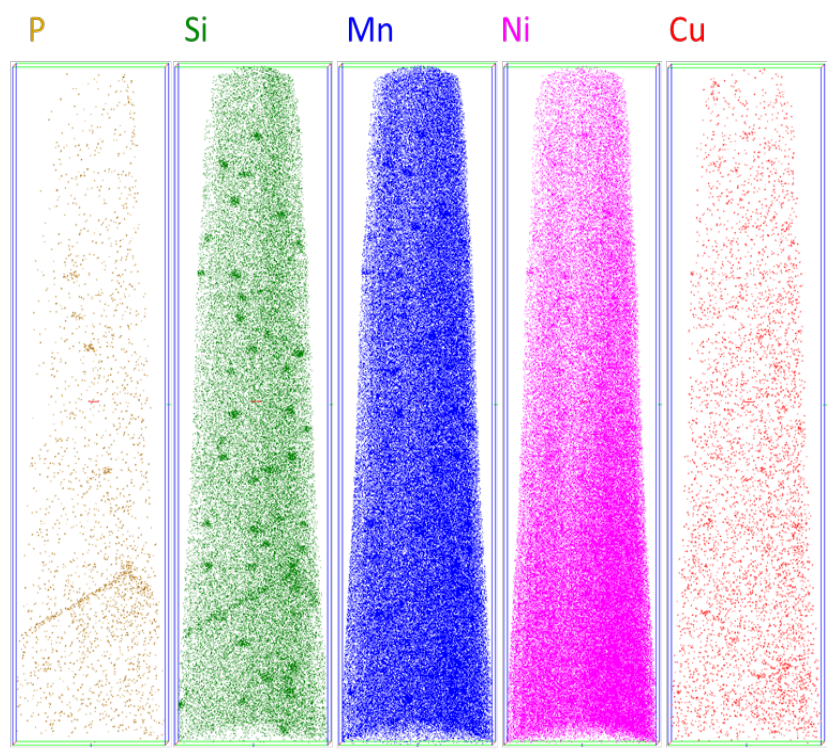

Fig 2 - Atom maps showing the distribution of solute atoms in a small volume of a typical French RPV steel (base metal) irradiated with neutrons at $290^{\circ} \mathrm{C}$ [14]. The sample has a volume of $45 \times 45 \times 190 \mathrm{~nm}^{3}$. 
Studies in model alloys for RPV steels using PAS techniques, suggest the existence of complexes with up to 10 vacancies associated with solutes [72-75]. This proves that the clusters depicted in Fig. 1i as a possible consequence of the assumptions of our model actually exist. Interestingly, PAS studies also reveal that the majority of vacancy-type defects are in fact found in a Fe-rich environment, i.e., they are not associated with a local high solute concentration. This can be appreciated by looking at $S-W$ curves, e.g. in Fig. 8 in Ref. [76]. It is seen that these curves, for irradiated RPV steels, are close to the one corresponding to pure Fe, and not to those associated with pure Ni or pure Mn materials. This indirectly suggests the dilute nature of the clusters against the compact shape that would be expected for nuclei of specific thermodynamic phases.

Fig. 3 summarizes the characteristics of the NSRC as seen by APT, for all cases listed in Tab. 1. It reveals no clear trends between density/size of NSRC and radiation dose, except for a weak tendency to slowly increase the number density and the volume fraction with dose, as is substantiated by a power law fit. The number density is generally measured between $10^{22} \mathrm{~m}^{-3}$ and $10^{23} \mathrm{~m}^{-3}$ in the whole range of doses from 0.01 to almost $1 \mathrm{dpa}$. The mean size of the NSRC lies between 1 and $4 \mathrm{~nm}$, with an average around $2.5 \mathrm{~nm}$ and essentially no increase with dose. Importantly, the volume fraction exhibits no sudden appreciable increase above a certain incubation dose. This suggests that the mechanism that is responsible for the formation of NSRC deviates from a classical nucleation and growth process: solute clusters keep more or less constant size, as if solutes were equally distributed in each of them, and slowly grow in number.
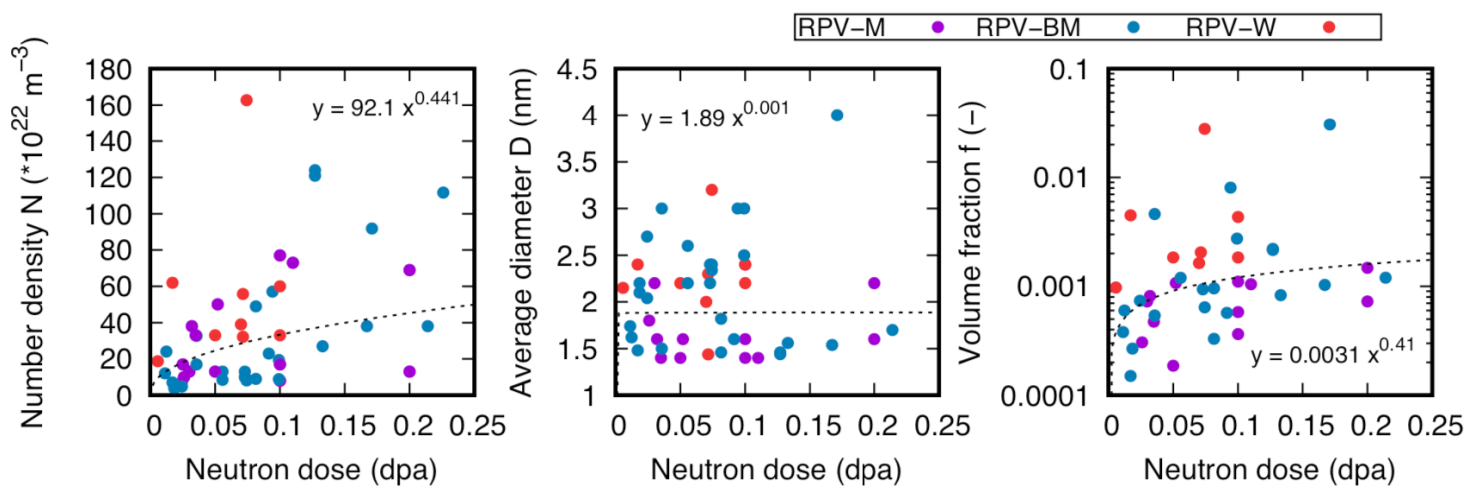

Fig. 3 - Evolution with the received neutron dose of the number density $N$, average size $D$ and volume fraction $\left(f=N D^{3} \pi / 6\right)$ of the NSRC, as evidenced by APT and SANS, and used as reference data in this work (see description and references in Tab. 1). Dashed lines show power law regressions.

Finally, experimental evidence for defects as depicted in Fig. 1h is found in the literature. The point-defect content of NSRC cannot be deduced by APT. Nevertheless, if the loop is large enough, due to the particularly favourable edge location, rings of solutes have been detected, for example toroidal segregations of $\mathrm{Mn}, \mathrm{Ni}$ and $\mathrm{Si}$ were observed in Russian VVER RPV base metals [71]. Coincidentally, dislocation loops were observed by TEM in the same materials [77], with densities $\left(\sim 10^{22} \mathrm{~m}^{-3}\right)$ and sizes (about $5 \mathrm{~nm}$ ) compatible with these toroidal APT solute clusters (respectively $6-7 \mathrm{~nm}$ and $10^{22} \mathrm{~m}^{-3}$ ). Similar APT observations of solute rings have been reported in [78]. Alternatively, lumps of solute clusters decorating discontinuously the loop edge line have been detected after careful analysis of APT results, as a system of close clusters lying on the same atomic plane [79]. Planar clusters of solute atoms were reported also in APT studies on VVER materials [13], 
as well as in austenitic steels (although in the latter case at a significantly higher dose, i.e., 10 to $28 \mathrm{dpa}$ ) [78]. These are all clear evidences that solute clusters are indeed associated with large loops in some irradiated steels, as stemming from the assumption of the model (Fig. 1h). Such clusters are, however, far less common than spherical clusters. To the authors' knowledge, they have never been observed in Western RPV materials, in the range of chemical compositions and irradiation conditions that are listed in Tab. 1. This is consistent with the physics of irradiation-induced defects, considering the differences of diffusion properties between SIA and vacancy defects: SIA defects tend to be onedimensionally migrating, thus the sink strength towards sinks diluted in the bulk of the materials is lower. Consequently, in Fig. 1 , JV is, in a majority of cases, higher than $\mathrm{J}_{\mathrm{I}}$. 
Table 1 - List of chemical composition and irradiation conditions for the RPV materials investigated in this study. The last column indicates the range of numerical values for the $E_{\text {Sat }}^{(\text {bind) }}$ parameter (defined in the supplementary material) that maximizes the agreement between OKMC simulations and experimental data. The abbreviations LDR, MDR and HDR stand for "low/medium/high neutron dose rate", respectively. Data denoted as "this work" were obtained during the LONGLIFE European Project [71].

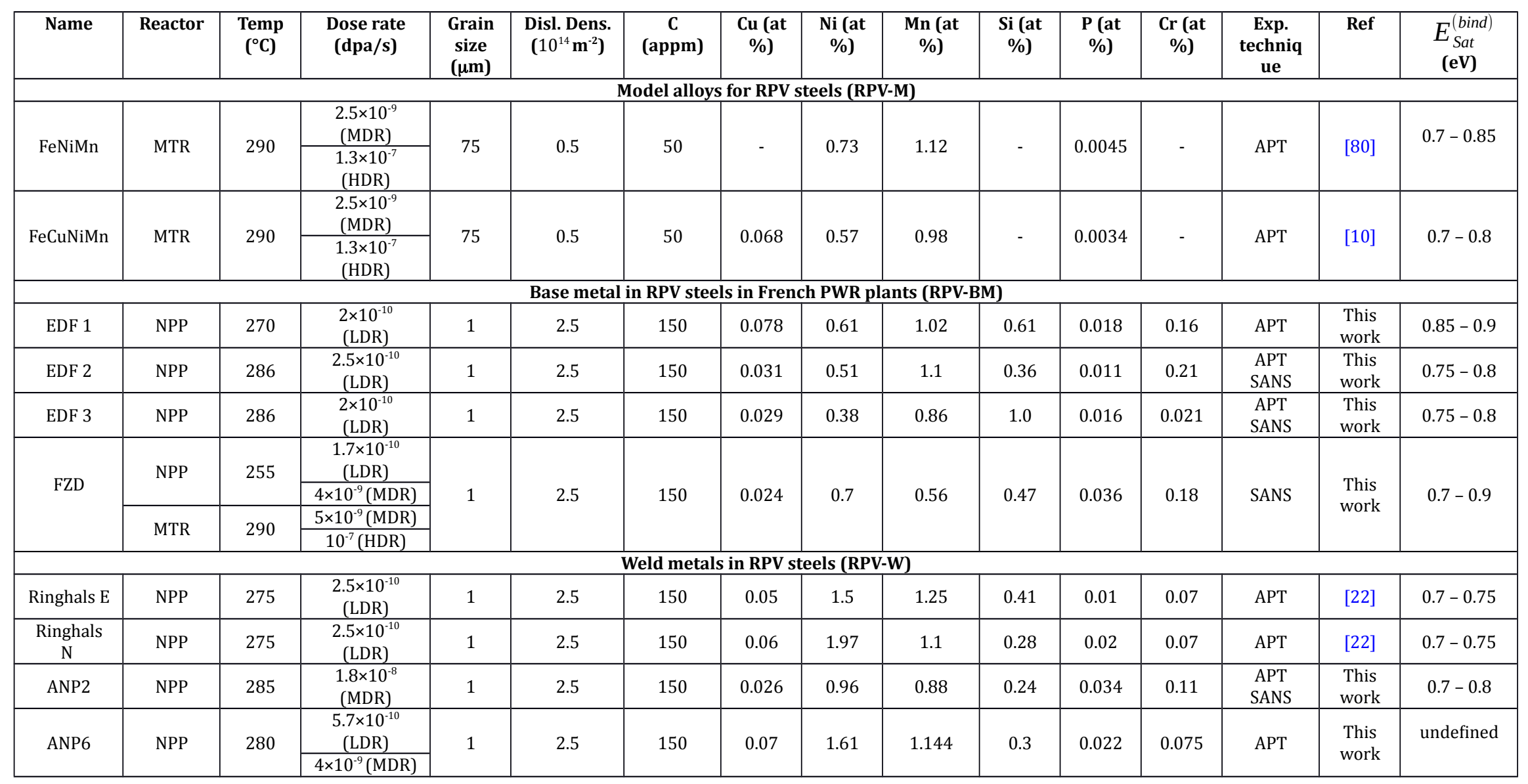




\section{Object kinetic Monte Carlo model for NSRC formation}

The relative importance of the different mechanisms summarized in Fig. 1 to drive the process of NSRC formation, can be quantified by introducing them, with suitable parameters, in a model that describes the evolution of the nanostructure. The features of this model, conceptually described in section 2 , are presented in what follows. Premises for such model were already proposed in previous work by Jansson et al. [81] and Chiapetto et al. [60-61], based on the object kinetic Monte Carlo (OKMC) method [82], but limiting the scope to simple model alloys (Fe-C, Fe-MnNi). Here we generalise those models, by adding all the required reactions for the mechanisms shown in Fig. 1, so as to describe neutron irradiation in steels (section 3.1), including explicit treatment of solute transport. This generalization is made possible by the massive calculation of parameters performed using DFT, as reported in section 3.2.

\subsection{General description of the model}

In an OKMC simulation, the evolution of a system that contains diffusing species is described stochastically. These can be point defects, their clusters, or complexes involving point defects and solutes - e.g. vacancy-solute clusters - altogether denoted as 'objects'. Each object is located at given coordinates in a simulation volume with periodic boundary conditions and has an associated reaction volume, conveniently assumed to be spherical, whenever this approximation is reasonable. Large loops have an associated toroidal volume. If mobile, the object can migrate by discrete jumps of a given distance, according to pre-defined probabilities. When the reaction volume of an object overlaps with the volume of another one, depending on the nature of the two, they may recombine (e.g. a self-interstitial with a vacancy), cluster (progressively forming cavities or dislocation loops), become trapped (if an immobile complex is formed), or be absorbed by sinks. Objects can also dissociate (e.g. by emission of a point defect) or move away from a trap, again according to pre-defined probabilities. All these are events. The probability for migration and dissociation events is expressed in terms of Arrhenius frequencies for thermally activated processes [83], in the framework of the transition state theory:

$$
\Gamma_{e}=v_{e} \exp \left(\frac{-E_{e}^{(a c t)}}{k_{B} T}\right)
$$

Here $v_{e}$ is the attempt frequency (or pre-factor) of the event $e$, and $E_{e}^{(a c t)}$ is the corresponding activation energy, both these parameters being an input to the model; $k_{B}$ is Boltzmann's constant and $T$ is the irradiation temperature expressed in K. Since the activation energy for migration or emission depends on the type and the size of the objects involved, a large number of parameters is needed for a standard simulation. These parameters may be derived using data from $a b$ initio calculations and/or molecular dynamics, as well as other atomistic calculations, or from experiments whenever available. In some cases, rate theory helps to extend them to larger cluster sizes, while sometimes educated guesses are unavoidable. The parameters used in this work for the properties of vacancies and self-interstitial clusters are the result of years 
accumulating information and addressing increasingly complex alloys, as detailed in [60$61,81]$. Departures from those parameters, or additions, are described in what follows.

The main and crucial addition introduced here with respect to previous work [60-61, 81] concerns the explicit treatment and redistribution of solute atoms of different chemical nature during irradiation. The work of Chiapetto et al. [60-61] considered the Fe-C-NiMn alloy. The effect of all alloying elements was accounted for in a 'grey alloy' approximation, i.e. their effect was translated into a change of the value of the parameters that define the mobility and stability of point defects and their clusters, as a function of the nominal composition: the effect was thus equal everywhere in the simulation volume and no solute redistribution could take place, because solutes were physically not in the box, only their effect. Here, in contrast, the Fe-C-CuNiMnSiP system is directly addressed, including explicitly all the alloying elements of steels used in nuclear applications that are known to produce NSRC. Doing this increases the complexity of the model, because the interaction of each solute with the other solutes and with point-defects needs to be described.

The way the model works is pictorially illustrated in Fig. 4.

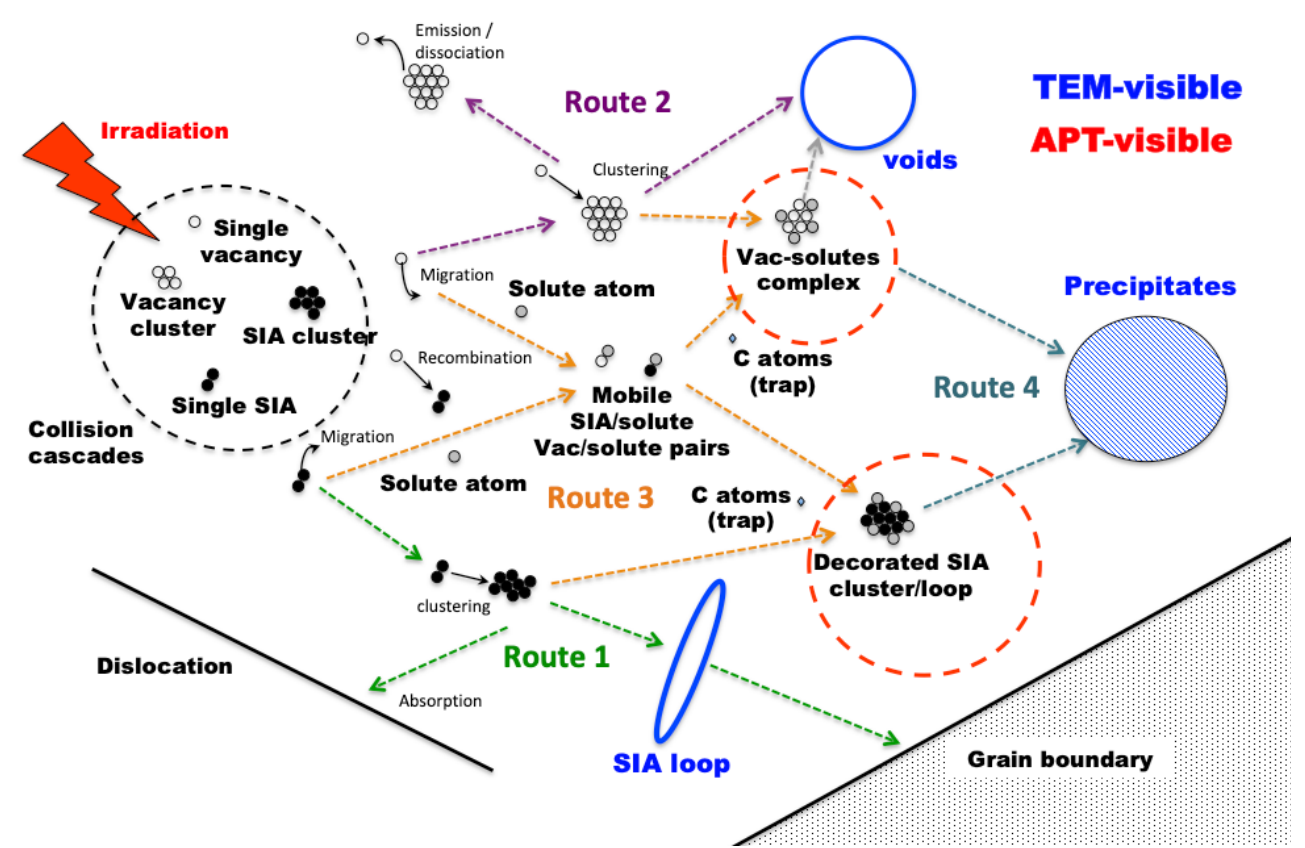

Fig 4 - Schematic description of our OKMC model. Different routes for the injected defects are highlighted with different colours, and so are the defects visible to the TEM or the APT experimental techniques.

The initial condition is a simulation volume devoid of point defects and their clusters. Substitutional solutes, namely $\mathrm{Cu}, \mathrm{Ni}, \mathrm{Mn}, \mathrm{P}$, and $\mathrm{Si}$, are randomly distributed in the volume, while $\mathrm{C}$ impurities are included as trapping objects (see detailed explanations in Refs. [60-61, 81, 84]), according to the bulk composition of the steel of interest. The dislocation network is represented by spherical sinks for point defects, with strength equivalent to the dislocation density observed in the material before irradiation. The grain size is accounted for by associating each object with two sets of coordinates: those in the simulation volume and those with respect to the centre of a grain. For convenience grains are treated as spheres. Periodicity is applied to the former set of coordinates, but not to the latter. Each newly created object is assigned a random position within the 
grain. When the position of the object coincides with the spherical surface (grain boundary), the object disappears.

Irradiation events, i.e. the appearance of a displacement cascade or the production of isolated Frenkel pairs (vacancy + self-interstitial), take place in a stochastic manner, based on the dose rate assigned, expressed in displacements-per-atom per second (dpa/s), applying the Norgett-Robinson-Torrens (NRT) equation [85, 86] (this choice is made to allow comparison with the dpa as calculated in experiments). A displacement cascade is, from the point of view of the model, a pre-defined list of point defects and their clusters, with defined positions with respect to the centre of mass of the cascade. Cascades are taken randomly from a database produced using molecular dynamics. Each is randomly rotated and injected into a randomly selected area of the simulation volume, as depicted in the left corner in Fig. 4. The thermally activated diffusion of the defects then takes place, as the Monte Carlo algorithm selects migration events. Some correlated recombination (i.e. within the cascade region) is likely to occur, but most surviving defects will diffuse to longer distances, following different possible routes (referring to Fig. 4):

- Route 1: SIA defects migrate in first instance because, in the absence of a significant concentration of solutes or impurities, their migration energy is low: $<0.35-0.5 \mathrm{eV}$ for three-dimensionally (3D) migrating single SIAs or small clusters thereof, and as low as $\sim 0.1 \mathrm{eV}$ or less for one-dimensionally (1D) gliding loops. 1D gliding loops may become trapped, or annihilate with vacancies, but many will leave the original cascade region and reach dislocations or grain boundaries. A small proportion of them, via clustering or trapping and coalescence, is expected to form bigger defects, becoming dislocation loops visible to TEM if they grow large enough $(>2 \mathrm{~nm}$ in diameter).

- Route 2: vacancy defects follow analogue fates as SIA defects in route 1, with the significant differences that: (a) the migration energy is larger $(>0.6 \mathrm{eV}$ and $>1 \mathrm{eV}$ for sizes above 5 vacancies) and therefore the diffusion kinetics is orders of magnitude slower; (b) the migration has a full 3D character instead of a 1D character, favouring interactions with the other defects in their immediate vicinity. Consequently, vacancy defects spontaneously form a higher density of smaller-sized defects. Nevertheless, some of them may eventually grow to form nanovoids, visible by TEM (>1.5 nm). Very few vacancies are expected to reach the grain boundary or dislocations.

Routes 1 and 2 deliberately describe radiation-induced nano-feature evolutions that take place in a relatively pure material, virtually free of solute atoms and containing essentially only $\mathrm{C}$ atoms or, to a lesser extent, other interstitial impurities, such as $\mathrm{N}$ and 0 , that act as traps forming complexes with vacancies [53]. The presence of solutes substantially reduces the mobility of defects (especially SIA):

- Route 3: Solute atoms, initially distributed in random positions in the matrix, strongly interact with migrating point defects or clusters. While the stable SIA-solute or vacancy-solute pairs that form are mobile, small SIA loops and vacancy clusters are rapidly immobilized by interacting with multiple solutes. Immobilized pointdefect clusters become sinks for point defects that drag solutes, thereby providing nucleation sites for NSRC. Depending on the flux of incoming point-defects and species, and on the stability of the point-defect/solute complexes that form, the NSRC can either grow or dissolve. Since they are immobile, coalescence hardly ever occurs, thereby preventing the formation of significantly larger features: this is likely to be the reason why voids or loops are only rarely observed in RPV steels by TEM, or only at sufficiently high dose. 
- Route 4: The NSRC formed in route 3, due to their small size (and often undefined thermodynamic phase nature) remain coherent with the lattice structure of the Fe matrix. Occasionally they may grow to such an extent that a local phase change may happen. One example could be the formation of an fcc $\mathrm{Cu}$ precipitate in $\mathrm{Fe}$, which experimentally only takes places when a coherent $\mathrm{Cu}$ cluster has grown above $5 \mathrm{~nm}$ diameter [87].

The initial conditions coupled with the parameters describing migration and dissociation events, together with the reaction volumes, will determine the resultant recombination, clustering, trapping and absorption rates in the simulation. If the physical parameterization is reasonable, the model will prove to be physically sound and accurately reflect the imposed operating conditions (temperature, dose-rate, dose, ...). For instance, in the range of $1-3$ at $\%$ of solutes, route 3 is expected to dominate, while route 1 and route 2 will dominate in very dilute alloys.

In the model presented here, the physical parameterization is based on an extensive database of migration and binding energy values calculated by DFT. However, this database cannot cover all possible combinations of solutes and point-defects, even less all size ranges. So, a simplified description of the binding energy values to extrapolate to all cluster sizes is needed. The parameters will also be affected by the initial conditions. Parameters such as the actual initial dislocation density or concentration of carbon atoms in the matrix are often not known, or known with low precision. In these cases, the values assigned to the initial conditions are more of an assumption than an actual experimental input. Unless APT measurements are available, uncertainties affect also the concentration in the matrix of solutes that form carbides or other phases during fabrication, for example Mn. Changes on these initial concentrations of solute atoms in the matrix may have an effect on the simulation results. A full range sensitivity study should be performed for all initial condition parameters; however, this would be extremely cumbersome and out of the scope of the present work, that does not have the ambition of presenting a fully calibrated model. Instead, we aim to demonstrate that the physical assumptions of the model lead to reasonable results based on comparison with experimental data. We thus use sensible parameters to evaluate which, out of all those described in section 2.1, are the dominant evolution pathways. Therefore, initial conditions such as dislocation density or $\mathrm{C}$ content of the matrix are here based on either known experimental data or reasonable guesses, and are the same for all steels, distinguishing only the case of model alloys, where lower $\mathrm{C}$ contents and dislocation densities than in steels are expected.

\subsection{Parameterization of events related to point-defect/solute clusters}

Four new events must be adequately parameterized in the OKMC model, for describing the interactions between solute atoms and point-defects, as depicted in Fig. 5. 

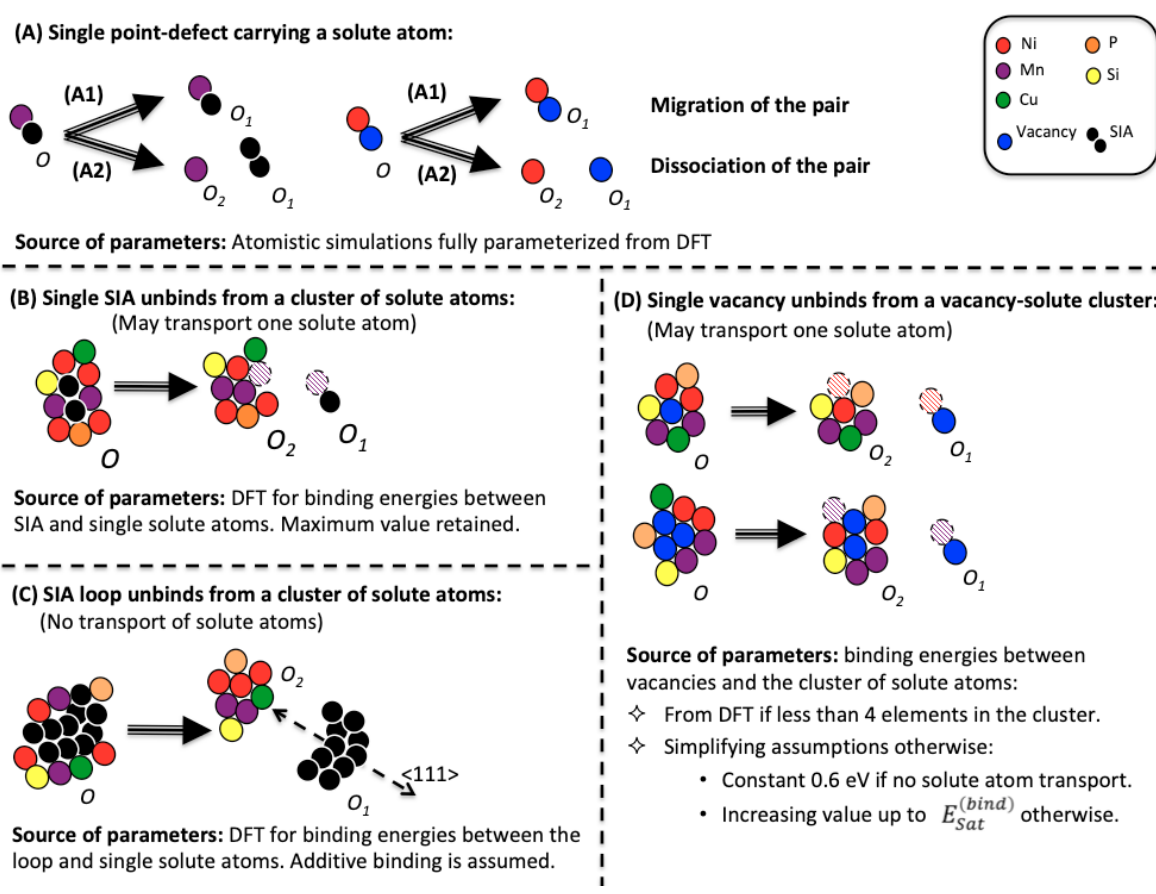

Fig. 5 - Definition of four events for an accurate description of the interactions between solute atoms and point-defects in our OKMC model.

Except for A1, all these events correspond to the separation of a reactant in two products, denoted as $O \rightarrow O_{1}+O_{2}$, where by convention $O_{1}$ is the object that migrates away from $\mathrm{O}_{2}$. In order to parameterize the events in Fig. 5 in an OKMC model, their frequency $\Gamma_{e}^{\square}$ in Eq. 1 needs to be calculated with an activation energy expressed, in general, as:

$$
E_{e}^{(a c t)}=E_{O_{1}}^{(m i g)}+E_{O_{1}, O_{2}}^{(b i n d)}
$$

Here, $e=\mathrm{A}, \mathrm{B}, \mathrm{C}$ or $\mathrm{D}$ in Fig. 5. The first term is the migration energy of $O_{1}$ alone and the second one is the binding energy between $O_{1}$ and $O_{2}$. The values of $E_{O_{1}, O_{2}}^{(\text {bind }}$ have been here calculated by DFT methods for a large number of small clusters, up to triplets. The use of DFT is necessary because no interatomic potentials [88] exist that can treat the required chemical complexity, nor are other cohesive energy models such as DFT-fitted cluster expansions [89] currently available. The shortcoming of using DFT is that the binding energy $E_{O_{1}, O_{2}}^{(b i d)}$ could not be systematically evaluated for all (or many) sizes, because of the prohibitive computing time that the evaluation of the combinatorially large number of possible NSRC chemical compositions would demand. Therefore, the binding energies associated with bigger defects than those tabulated in the supplementary material must be estimated:

$$
\begin{aligned}
E_{O_{1}, O_{2}}^{(b i n d)} & =\left.E_{O_{1}, O_{2}}^{(b i n d)}\right|_{D F T} & \text { if available } \\
& =\left.E_{O_{1}, O_{2}}^{\text {bind })}\right|_{E s t} & \text { otherwise }
\end{aligned}
$$


Here, the subscript "Est" refers to cases where the binding energy is estimated by extrapolating from the available DFT data, using simplifying assumptions that are described in detail in the supplementary material.

The main assumption in our model concerns event $\mathrm{D}$, which describes the dissociation of a single vacancy, or a vacancy-solute pairs, from a NSRC. For simplicity, we estimated the binding energy between a NSRC and a single vacancy assuming that $E_{O_{1}, O_{2} \mid \text { Est }}^{\text {bind }}$ in Eq. 4 has a constant value of $0.6 \mathrm{eV}$. In contrast, the binding energy with a vacancy-solute pair was assumed to increase with the size of the NSRC, expressed in terms of the absolute number of solute atoms in the defect. Inspired from our database of DFT data, we assumed that in this case $\left.E_{O_{1}, O_{2}}^{\text {bind }}\right|_{\text {sst }}$ linearly increases from $0.52 \mathrm{eV}$ until the NSRC contains 65 atoms. It is then assumed to have a constant, saturated value, henceforth denoted as $E_{\text {Sat }}^{(b i n d)}$.

\subsection{Comparison of OKMC predictions with APT}

In our OKMC model a NSRC is represented by a composition vector (see supplementary material) assigned to a specific location in space. To compare with APT, this representation must be converted into an emulation of the reconstructed 3D data, as observed by the experimental technique. This is done in two steps, as illustrated in Fig. 6 . As a first step, an atomic configuration needs to be created by distributing the solute atoms and the point defects in a confined region of space around the position of the cluster. To do this we deliberately assume that the solute atoms are arranged in a compact manner, filling all neighbouring positions around the central coordinates. Fe atoms are also included in the spherical cluster, in randomly chosen positions, corresponding to the number of solute-free dumbbells that were absorbed by the clusters. The second step consists of treating the data to emulate the experimental limitations associated with APT, thereby enabling a like for like comparison between simulation and experiment. During an APT experiment individual atoms are field evaporated from the sample and their positions of impact on a detector are used to determine their relative positions prior to evaporation. The process is not perfect since not all of the atoms are detected (typically between $37 \%$ and $60 \%$, depending on the equipment) and the field evaporation is extremely complex, leading to inherent artefacts such as a degraded spatial resolution and trajectory aberrations. The detection efficiency is straightforward to emulate, namely atoms from model data are eliminated at random to yield the appropriate detection efficiency. For simplicity, even though experiments were performed with different equipment, we assume a constant detection efficiency of $40 \%$ for all chemical species. The position uncertainty is greater in the lateral direction with respect to the normal of the surface of the APT needle, than longitudinally [90] and can be sufficiently large to prevent resolution of extremely small clusters. To simulate this effect in the model data, a Gaussian distributed random noise (FWHM=1nm in the plane perpendicular to the needle axis, FWHM=0.1nm in the direction of the needle axis) has been applied to the atomic coordinates [90]. Finally, a simple close neighbours algorithm is applied in order to search for clusters in the simulation box. It has to be noticed that artefacts such as trajectory focusing are not explicitly considered here. The analysis of the simulation results, after the treatment that is described above, is less critical than the analysis of experimental APT data, because in fact precise information is 
available about every single atom: the randomization as described is introduced to be more consistent with the situation of the experiment. Importantly, the simulation volumes are comparable with usual APT tip volumes, i.e. $-10^{5}-10^{6} \mathrm{~nm}^{3}$.

$\mathrm{ONi} \bigcirc \mathrm{MnO} \mathrm{Cu} O \mathrm{P} \mathrm{O} \mathrm{Si}$

(A)

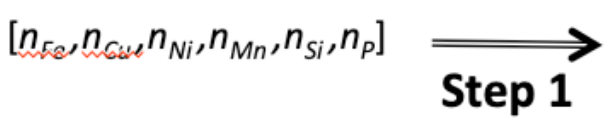

(B)

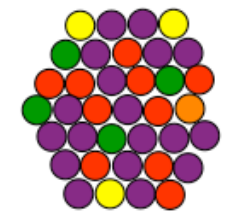

(C)

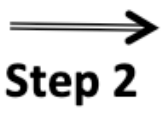

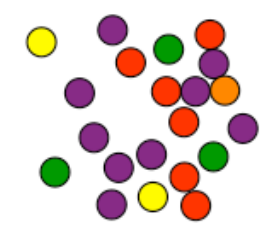

Fig. 6 - Procedure followed for the comparison between NSRC predicted as en outcome of OKMC predictions and experimental evidence with APT. On (A) the solute clusters as described in the OKMC model (composition vector) are transformed into a compact 3D structure (B), which are later on virtually distorted as the APT measurement would do (C).

\section{Application and results}

In this section, we apply the OKMC model described in section 3, to the whole set of materials and irradiation conditions listed in Tab. 1. A few input parameters deserve preliminary discussion:

- The chemical compositions given in the table are the measured matrix values from APT and they are used as input for the model. The values differ from the nominal chemical compositions specified by the steel manufacturers, because some solutes (for example $\mathrm{Mn}$ ) tend to form carbides. Where APT data was not available (e.g. when samples were only analysed with SANS), it was assumed that $20 \%$ of the Mn solutes was located in carbides [60-61].

- We assumed that the grain size is $75 \mu \mathrm{m}$ and the dislocation density is $5 \cdot 10^{13} \mathrm{~m}^{-2}$ for all model alloys [10, 7], which are ferritic materials, because in this case measurements exist. The ambiguous definition of the grain size in the bainitic phase and the often lacking assessment of the dislocation density before irradiation in the case of steels obliged us to assume a grain size of $1 \mu \mathrm{m}$ and a dislocation density of $2.5 \cdot 10^{14} \mathrm{~m}^{-2}$, based on only a few studies [91].

- The concentration of free $C$ in the bulk of the bainitic phase is rarely (if ever) reported, because experimentally this measurement is not trivial. Most $\mathrm{C}$ is expected to precipitate in carbides, or segregate at grain boundaries and dislocations, but some remains in the matrix. In this study, we adopt similar hypotheses to those in [60-61, 81, 84], namely the bulk $C$ concentration is assumed to be 50 appm for all model alloys and 150 appm in the case of steels.

The development of a model with fully quantitative capabilities ideally requires an accurate evaluation of the impact of all the above parameters that define the initial conditions. However, the numerical values chosen here can be considered realistic and will not change over orders of magnitude. Furthermore, in this study our purpose is not to obtain the best quantitative agreement possible for each case. Instead, we aim to 
evaluate whether the mechanisms that have been implemented in the model, as in Fig. 1, are sufficient to explain quantitatively the experimentally observed formation of NSRC in steels under irradiation, and if so which evolution pathways dominate.

The one parameter that is mainly responsible for inaccuracies is $E_{\text {Sat }}^{(b i n d)}$ defined above, because of the approximation that is made by assuming that a quantity that should depend on the local atomic configuration is a constant, irrespective of the accumulated dose, i.e. irrespective of the evolution of the size and composition of the NSRC. Different values were used for this parameter, to appreciate its impact on the model predictions.

Simulations were performed in boxes with dimensions $110 \mathrm{a}_{0} \times 120 \mathrm{a}_{0} \times 180 \mathrm{a}_{0}$, i.e., 31.6 $\mathrm{nm} \times 34.4 \mathrm{~nm} \times 51.7 \mathrm{~nm}$ which contain 4.752 million atoms. Each simulation was in fact performed in ten independent boxes, and overall statistics were performed; in each case, the total volume being analysed following the procedure of comparison with APT as described in section 3.3 was thus $5.62 \times 10^{5} \mathrm{~nm}^{3}$, containing 47.5 million atoms. The results are summarized in Fig. 7 to Fig. 10. The left panels compare the geometric features of the NSRC as predicted by our OKMC model with experimental evidence. The number density is affected by the numerical value of $E_{\text {Sat }}^{\text {bind })}$, while the average size is almost insensitive to it. Overall, one can see that the OKMC predictions fall in the experimental range, provided that the value of $E_{\text {Sat }}^{(b i n d)}$ is in an adequate range. Remarkably, the latter appears to be reasonably small and limited to:

$$
E_{\text {Sat }}^{(b i n d)}=0.7-0.9 \mathrm{eV}
$$

In specific steels the range of uncertainty is even narrower. Only a few cases exhibit a significant discrepancy with experimental evidence: (a) two materials, namely, the FeCuNiMn alloy at high dose-rate (in Fig. 7) and the FZD steel at medium dose-rate (in Fig. 8) show a large scatter of experimental data, which makes the comparison with the OKMC model more open to interpretation; (b) The ANP6 cases (in Fig. 10) were less satisfactorily predicted by the OKMC model.

Concerning the Fe content in the NSRC, our model predicts it to be in a range between $5 \%$ and $10 \%$, as reported in the extra figures in the supplementary material. This is however difficult to compare with experimental evidence, because APT data is likely to be influenced by inherent artefacts associated with this technique [33, 92]. For this reason, the central panels in Fig. 7 to Fig. 10 report the mean chemical compositions of the NSRC (normalized to 1 ) in terms of $\mathrm{Ni}, \mathrm{Mn}, \mathrm{Si}$ and $\mathrm{P}$, excluding the Fe content. The same was done for the APT data reported in the figures. One can see that the compositions of the NSRC in the OKMC model tend to correlate with the overall chemical composition of the material. This was expected, because the driving force for their formation in our model is the transport of matrix solute atoms towards sinks, irrespective of the composition of existing clusters. We see that the observations by APT appear to be in relatively good agreement with this result. The only clear exception is the FeCuNiMn model alloy. This is not surprising, considering that in this system strong thermodynamic driving forces are at work (Cu insolubility) which are not included in the present model.

Lastly, the right panels in Fig. 7 to Fig. 10 report the composition of the NSRC in term of point defects. Overall, OKMC predicts that $5 \%$ to $20 \%$ of them have a vacancy content. Likewise, no more than $20 \%$ to $50 \%$ are predicted to have an SIA content, while the remaining part is predicted to be free of point-defects. From a different point of view, extra figures provided in the supplementary material highlight that OKMC predicts that 
only $5 \%$ to $15 \%$ of the vacancies are associated with solute atoms. This is in good agreement with PAS evidence [72-75].

The analysis of the details of the events that take place during the OKMC simulations helps to rationalize the different mechanisms described in Fig. 1. Strictly speaking, making a detailed balance of each transition depicted in the figure is not straightforward, because they involve a large number of actual KMC events. Overall, an analysis of the output of the OKMC simulations reveals that a large proportion of NSRC has an SIA origin. Depending on the case (material, irradiation condition, etc), and the assumed value for $E_{\text {Sat }}^{\text {bind }}$, we estimate that $80 \%$ to $95 \%$ of the NSRC are originally nucleated by SIA defects, i.e., following the mechanisms depicted in Fig. 1a, Fig. 1b and Fig. 1c. This can be further illustrated by analysing the sensitivity of the model predictions with respect to the events defined in Fig. 5 (fully described in the supplementary material):

- Events A are the only source of solute transport. They are thus essential in the model, because no formation of NSRC would be predicted otherwise. It is worth noting that they are accurately parameterized from DFT calculated migration energies.

- Events B describe the interaction between single SIA defects and solute clusters. Our simulations reveal that these events are relatively rare, and they thus have a negligible influence on the model predictions.

- Events C describe the interaction between SIA loops and solute clusters. Our assumption is that the total binding energy is additive from contributions with individual solutes. This has the consequence that a loop is fully immobilized as soon as it is decorated by a couple of solute atoms. This is clearly a key assumption in the model. If $E_{O_{1}, O_{2}}^{(\text {bin })}$ is assumed to be negligible for events $\mathrm{C}$, then the OKMC model predicts a number density of NSRC about an order of magnitude lower.

- Events D describe the interaction between single vacancies and NSRC. The sensitivity of the model predictions with respect to the involved parameter $E_{\text {Sat }}^{(b i n)}$ is illustrated in Fig. 7 to Fig. 10. The lower $E_{\text {Sat }}^{(b i n d)}$, the lower the number density of NSRC predicted by the OKMC model. 

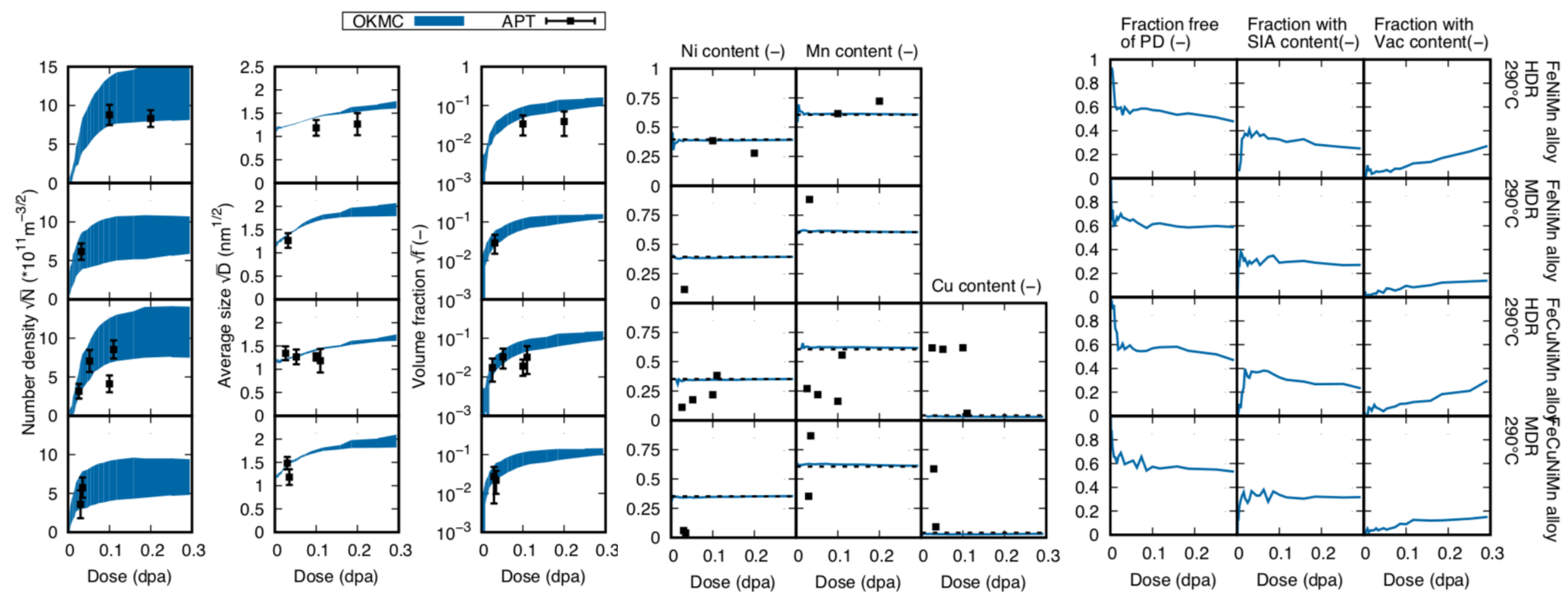

Fig. 7 - Summary of the prediction of our OKMC model for the formation of NSRC in model alloys, when irradiated in a MTR. Where possible, direct comparison is done with experimental evidence from APT and/or SANS. The line thickness for OKMC predictions denotes the uncertainty with respect to the saturated binding energy $E_{\text {Sat }}^{\text {bind }}$, as defined in the supplementary material. The left panels summarize the geometry of the NSRC in term of number density $N$, average size $D$, and volume fraction $\left(f=N D^{3} \pi / 6\right)$ in the material. The central panels summarize the chemical composition of the NSRC, normalized to unity and excluding Fe. Dashed horizontal lines indicate the content in the material, computed from Tab. 1. Finally, the right panels inform about the fraction of NSRC containing vacancies, SIA's, or no point-defects. 

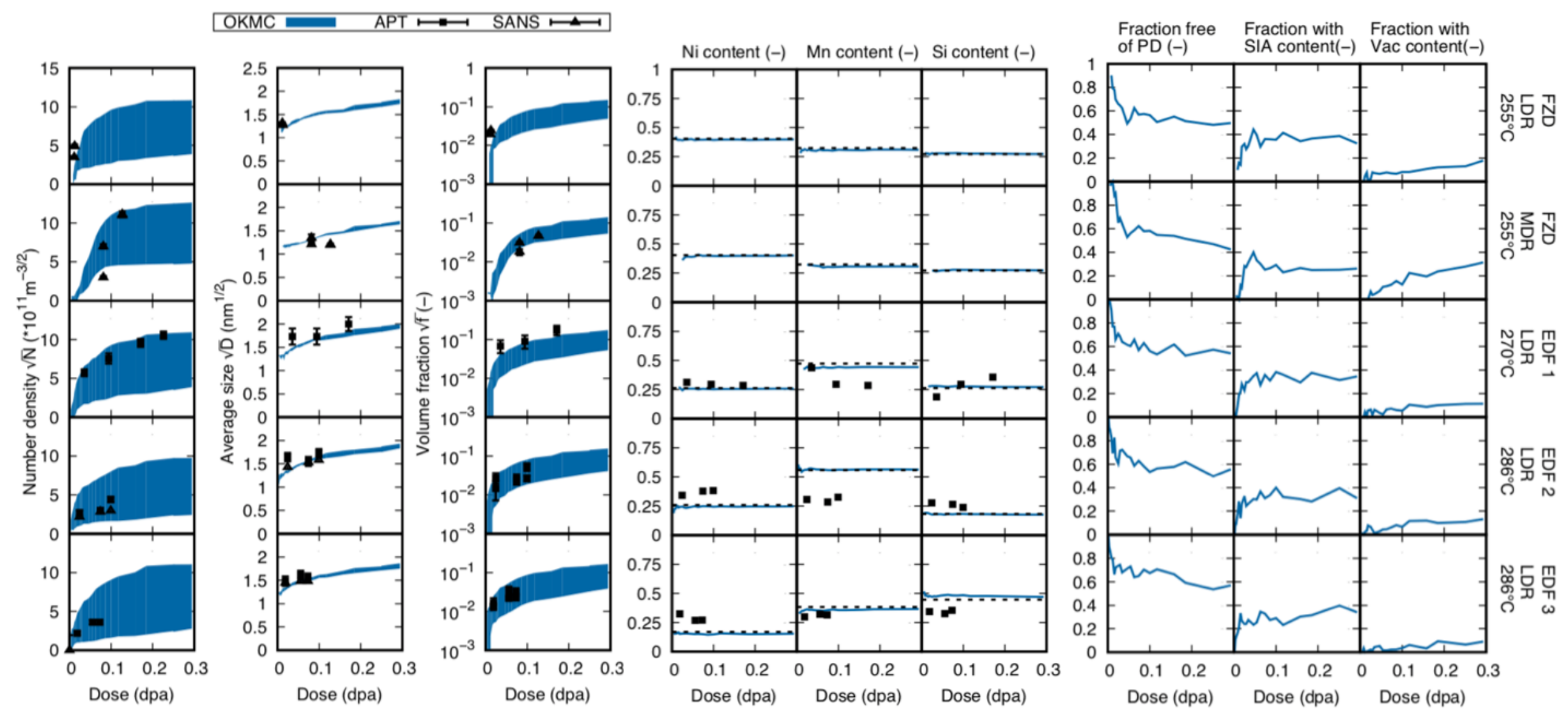

Fig. 8 - Summary of the prediction of our OKMC model for the formation of NSRC in base metals in RPV steels, when irradiated in PWR conditions. Legend is as in Fig. 7. 

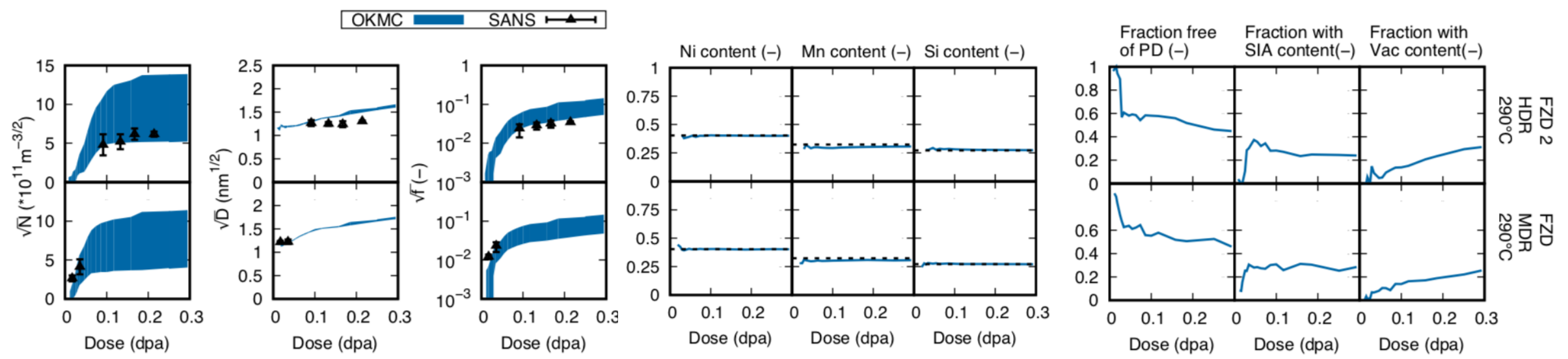

Fig. 9 - Summary of the prediction of our OKMC model for the formation of NSRC in base metals in RPV steels, when irradiated in a MTR. Legend is as in Fig. 7. 


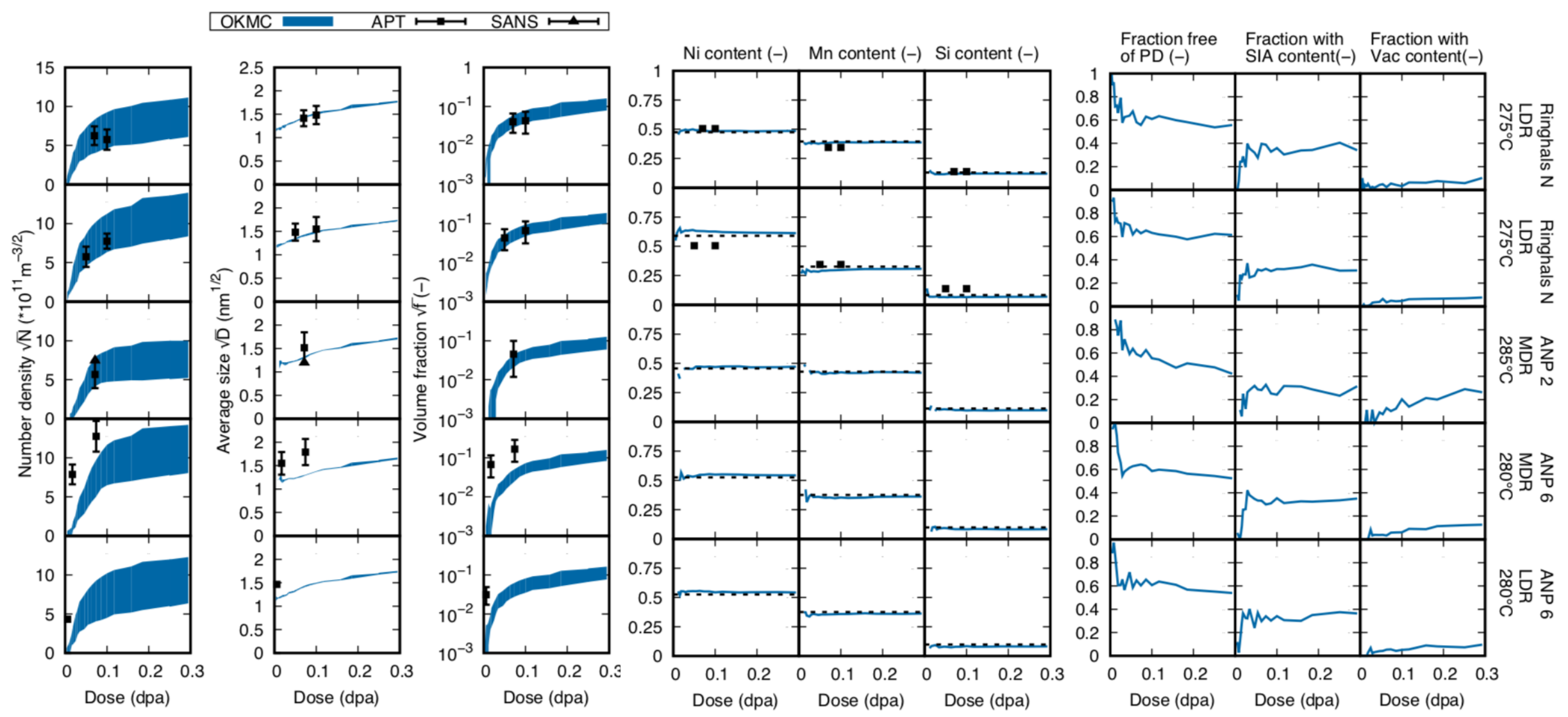

Fig. 10 - Summary of the prediction of our OKMC model for the formation of NSRC in weld metals of RPV steels, when irradiated in PWR conditions. Legend is as in Fig. 7. 


\section{Discussion and conclusion}

Our model provides reasonable predictions of the nanostructure evolution under irradiation, as compared with post-irradiation APT and SANS examination results, in a fairly wide range of alloy compositions and irradiation conditions. This suggests that the physical mechanisms depicted in Fig. 1, on which it is based, are realistic. The key mechanisms are thus: (a) dragging of solute atoms by single point-defects; (b) heterogeneous nucleation of solute clusters on small point-defects cluster, especially SIA clusters, as revealed while analysing the results of our simulations. Strictly speaking what is depicted in Fig. 1 is not precipitation, but solute segregation at sinks. This is a consequence of the processes strongly supported by DFT calculations and atomistic simulations. The composition of the clusters is thus largely the consequence of the transport of solutes by point defects. Therefore, depending on the respective diffusivities and thus on temperature, it should bear some correlation with the alloy composition. Importantly, in this scenario the fundamental mechanisms never change over the duration of the irradiation and the NSRC develop in a continuous way. This agrees with the absence of observation of changes of embrittlement mechanisms with increasing neutron dose [93].

The most simplifying assumption in our OKMC model is the use of a constant "saturated binding energy" (denoted as $E_{\text {Sat }}^{(\text {bind) }}$ above and defined in details in the supplementary material) between a point-defect/solute pair and a large solute cluster. This parameter is by nature local and should depend on the composition of the cluster (solutes and fraction of vacancies), but it is here assumed to be global and constant over time. Since the composition of the clusters depends on the composition of the steels, some variability of this parameter from material to material is physiological and unavoidable. This variability is indeed observed. The composition of the clusters may also be changing with dose, thus the assumption of a single constant value may fail even along the same simulation in a given steel. In some cases it does happen that a wider range of variation for $E_{\text {Sat }}^{\text {bind }}$ is needed to match the experimental data points. However, the variability is limited to a small range of values (see Eq. 5 and discussions above) and the assumption of a single constant value fails only in a few cases, thus making it an acceptable approximation to describe the process of vacancy emission and cluster dissolution. In any case, since this "saturated energy" has a clear physical definition, it can potentially be better assessed in the future, or even the approximation of a single parameter might be removed, by extending DFT calculations to larger sizes. This is currently not feasible because, in addition to computing time limitations, it entails a practical problem of data handling, as well as smart interpolation schemes, that could possibly be tackled in the future with the use of machine learning [94].

The fact that solute clusters can only dissolve after their SIA content has been removed suggests that a large proportion of clusters effectively observed should contain SIA, i.e. be associated with SIA clusters or loops. This is what our model predicts, as illustrated in the right panels in Fig. 7 to Fig. 10. While solute clusters will also continuously form on vacancy clusters without any SIA content, these will also be inherently less stable, because they can dissolve, thereby indeed surviving only if grown above a critical size, similarly to a 'nucleation and growth' process, and are proportionally less. These small, continuously forming and dissolving vacancy-solute clusters are unlikely to be seen by APT, due to their size, but may be identified by PAS, thanks to the chemical signatures in 
the environment around vacancies. Evidence of the formation of vacancy-Mn-Ni solute clusters from PAS has indeed been found [73-76]. It is unclear, however, whether these clusters can be associated with the so-called "unstable matrix damage" postulated as an additional hardening contribution at high neutron dose rate $[69,70]$, because it is unclear whether they will actually measurably contribute to hardening and ensuing embrittlement. MD simulations suggest that the presence of vacancies in the cluster reduces, rather than increasing, its strength [95], when interacting with edge dislocations. In contrast, when interacting with screw dislocations the formation of helical turns may result in a strong hindrance to dislocation motion [96]. It is difficult to anticipate which effect will be dominant.

In a recent work, Ke et al. [43] proposed a cluster dynamics model for RPV steels based on the underlying idea that irradiation mainly accelerates the kinetics of phase separation due to vacancy supersaturation. They thus inherently assumed that the NSRC are precipitates of thermodynamically stable phases, following the predictions of Thermo-Calc [67]. As a correcting factor, the model of Ke et al. also included an extra term generically associated with "radiation-induced heterogeneous nucleation of precipitates on point defect clusters produced in displacement cascades". Interestingly, the fitting of the model to experimental data revealed that the latter term is dominant in most cases over the thermodynamic component, in some cases by orders of magnitude. This result is thus in good agreement with the result of the present study. In spite of putting emphasis on two different aspects (thermodynamics driving forces or solute transport and heterogeneous seeding), both approaches therefore strongly suggest that SIA defects play a dominant role in NSRC formation and evolution under irradiation.

In perspective, the developed model could be applied to other alloys and steels, including ferritic/martensitic steels for next generation reactors. In terms of model improvement, sensitivity studies and application to simulate post irradiation annealing to assess the thermal stability of the nanostructural features are expected to be instrumental. When coupled to existing physical models for the assessment of radiation hardening [35-36], the present model is expected to provide a power toll for the assessment of radiation-induced hardening in irradiated RPV steels under a variety of irradiation conditions, including conditions of relevance for long term operation, for which experimental data are limited.

\section{Acknowledgements}

This work has received partial funding from the Euratom research and training programme 2014-2018 under Grant Agreement No. 661913 (SOTERIA project: Safe long-term operation of light water reactors based on improved understanding of radiation effects in nuclear structural materials) and Grant Agreement No. 249360 (LONGLIFE project: Treatment of long term irradiation embrittlement effects in RPV safety assessment). The views and opinions expressed herein do not necessarily reflect those of the European Commission. 


\section{References}

[1] P. Petrequin, A review of formulas for predicting irradiation embrittlement of reactors vessel materials, AMES Report No. 6, EUR 16455, European Commission, Luxembourg (November 1996).

[2] C. English, J. Hyde, Radiation damage of reactor pressure vessel steels, in: R.J.M. Konings (Editor-in-chief), Comprehensive Nuclear Materials Vol. 4: Radiation effects in structural and functional materials for fission and fusion, Elsevier Ltd., 2012, pp. 151-180.

[3] W.L. Server, R.K. Nanstad, G.R. Odette, Use of reactor pressure vessel surveillance materials for extended life evaluations using power and test reactor irradiations. Third International Conference on Nuclear Power Plant Life Management (PLiM) for Long Term Operations (LTO), International Atomic Energy Agency (IAEA), 2012.

https://inis.iaea.org/collection/NCLCollectionStore/_Public/43/070/43070853 .pdf

[4] A. Ulbricht, E. Altstadt, F. Bergner, H.-W. Viehrig, U. Keiderling, Small-angle neutron scattering investigation of as-irradiated, annealed and reirradiated reactor pressure vessel weld material of decommissioned reactor, J. Nucl. Mat. 416 (2011) 111-116.

[5] R. Chaouadi, R. Gérard, Neutron flux and annealing effects on irradiation hardening of RPV materials, J. Nucl. Mater. 418 (2011) 137-132.

[6] P. Efsing, J. Roudén, P. Nilsson, Flux effects on radiation induced aging behaviour of low alloy steel weld material with high nickel and manganese content, in: M. Kirk, E. Lucon (Eds.), Effects of Radiation on Nuclear Materials: 26th Volume, ASTM International, West Conshohocken, 2014, pp. 119-134. https://doi.org/10.1520/STP157220130112.

[7] L. Malerba, E. van Walle, C. Domain, S. Jumel, J.-C. van Duysen, State of advancement of the International REVE Project: Computational Modelling of Irradiation-Induced Hardening in Reactor Pressure Vessel Steels and Relevant Experimental Validation Programme. Proceedings $10^{\text {th }}$ Intl. Conference on Nuclear Engineering (ICONE-10), Arlington, VA, USA, April 14-18, 2002, The American Society of Mechanical Engineers, 2002, pp. 267-274.

[8] J.-P. Massoud, S. Bugat, B. Marini, D. Lidbury, S. van Dyck, PERFECT - Prediction of Irradiation Damage Effects on Reactor Components: A summary, J. Nucl. Mater. 406 (210) 2-6.

[9] A. Al-Mazouzi, A. Alamo, D. Lidbury, D. Moinereau, S. van Dyck, PERFORM 60: Prediction of the effects of radiation for reactor pressure vessel and in-core materials using multi-scale modelling - 60 years foreseen plant lifetime, $\mathrm{Nucl}$. Eng. Design 241 (2011) 3403-3415.

[10] E. Meslin, B. Radiguet, P. Pareige, A. Barbu, Kinetic of solute clustering in neutron irradiated ferritic model alloys and a French pressure vessel steel investigated by atom probe tomography, J. Nucl. Mater. 399 (2010) 137-145.

[11] P. Auger, P. Pareige, M. Akamatsu, D. Blavette, APFIM investigation of clustering in neutron-irradiated Fe-Cu alloys and pressure vessel steels. J. Nucl. Mater. 225 (1995) 225-230.

[12] P. Auger, P. Pareige, S. Welzel, J.-C. van Duysen, Synthesis of atom probe experiments on irradiation-induced solute segregation in French ferritic pressure vessel steels. J. Nucl. Mater. 280 (2000) 331-344. 
[13] M.K. Miller, K.F. Russell, J. Kocik, E. Keilova, Embrittlement of low copper VVER 440 surveillance samples neutron-irradiated to high fluences. J. Nucl. Mater. 282 (2000) 83-88.

[14] K. Fukuya, K. Ohno, H. Nakata, S. Dumbill, J.M. Hyde, Microstructural evolution in medium copper low alloy steels irradiated in a pressurized water reactor and a material test reactor. J. Nucl. Mater. 312 (2003) 163-173.

[15] P. Pareige, R.E. Stoller, K.F. Russell, M.K. Miller, Atom probe characterization of the microstructure of nuclear pressure vessel surveillance materials after neutron irradiation and after annealing treatments, J. Nucl. Mater. 249 (1997) 165-164.

[16] H. Huang, B. Radiguet, P. Todeschini, G. Chas, P. Pareige, Atom Probe Tomography characterization of the microstructural evolution of a low coper reactor pressure vessel steel under neutron irradiation. Mater. Res. Soc. Symp. Proc. 1264 (2010) BB05-18.

[17] M.K. Miller, A.A. Chernobaeva, Y.I. Shtrombakh, K.F. Russell, R.K. Nanstad, D.Y. Erak, 0.0. Zabusov, Evolution of the nanostructure of VVER-1000 RPV materials under neutron irradiation and post irradiation annealing, J. Nucl. Mater. 385 (2009) 615-622.

[18] M.K. Miller, K.F. Russell, Embrittlement of RPV steels: An atom probe tomography perspective. J. Nucl. Mater. 371 (2007) 145-160.

[19] B.A. Gurovich, E.A. Kuleshova, Ya.I. Shtrombakh, D.Yu. Erak, A.A. Chernobaeva, 0.0. Zabusov, Fine structure behaviour of VVER-1000 RPV materials under irradiation, J. Nucl. Mater. 389 (2009) 490-496.

[20] M.K. Miller, K.F. Russell, M.A. Sokolov, R.K. Nanstad, Atom probe tomography characterization of radiation-sensitive KS-01 weld.J. Nucl. Mater. 320 (2003) 177-183.

[21] E. Meslin, M. Lambrecht, M. Hernández-Mayoral, F. Bergner, L. Malerba, P. Pareige, B. Radiguet, A. Barbu, D. Gómez-Briceño, A. Ulbricht, A. Almazouzi, Characterization of neutron-irradiated ferritic model alloys and a RPV steel from combined APT, SANS, TEM and PAS analyses, J. Nucl. Mater. 406 (2010) 73-83.

[22] M.K. Miller, K.A. Powers, R.K. Nanstad, P. Efsing, Atom probe tomography characterizations of high nickel, low copper surveillance RPV welds irradiated to high fluences. J. Nucl. Mater. 437 (2013) 107-115.

[23] P.D. Edmondson, M.K. Miller, K.A. Powers, R.K. Nanstad, Atom probe tomography characterization of neutron irradiated surveillance samples from the R. E. Ginna reactor pressure vessel, J. Nucl. Mater. 470 (2016) 147-154.

[24] R.G. Carter, N. Soneda, K. Dohi, J.M. Hyde, C.A. English, W.L. Server, Microstructural characterization of irradiation-induced $\mathrm{Cu}$-enriched clusters in reactor pressure vessel steels, J. Nucl. Mater. 298 (2001) 211-224.

[25] P.A. Beaven, F. Frisius, R. Kampmann, R. Wagner, J.R. Hawthorne, SANS investigation of irradiated A533-B steels doped with phosphorus, in: L.E. Steele (Ed.), Radiation embrittlement of nuclear reactor pressure vessel steels: An international review, ASTM STP 1011, ASTM International, West Conshohocken, 1989, pp. 243-256. https://doi.org/10.1520/STP10399S

[26] M.K. Miller, B.D. Wirth, G.R. Odette, Precipitation in neutron-irradiated $\mathrm{Fe}-\mathrm{Cu}$ and $\mathrm{Fe}-\mathrm{Cu}-\mathrm{Mn}$ model alloys: a comparison of APT and SANS data, Mater. Sci. Eng. A353 (2003) 133-139. 
[27] F. Bergner, M. Lambrecht, A. Ulbricht, A. Almazouzi, Comparative small-angle neutron scattering study of neutron-irradiated Fe, Fe-based alloys and a pressure vessel steel, J. Nucl. Mater. 399 (2010) 129-136.

[28] A. Wagner, A. Ulbricht, F. Bergner, E. Altstadt, Influence of the copper impurity level on the irradiation response of reactor pressure vessel steels investigated by SANS, Nucl. Instr. Meth. Phys. Res. B 280 (2012) 98-102.

[29] F. Bergner, C. Pareige, M. Hernández-Mayoral, L. Malerba, C. Heintze, Application of a three-feature dispersed-barrier hardening model to neutron-irradiated FeCr model alloys, J. Nucl. Mater. 448 (2014) 96-102.

[30] M. Lambrecht, E. Meslin, L. Malerba, M. Hernández-Mayoral, F. Bergner, P. Pareige, B. Radiguet, A. Almazouzi, On the correlation between irradiationinduced microstructural features and the hardening of reactor pressure vessel steels, J. Nucl. Mater. 406 (2010) 84-89.

[31] Z. Lu, R. Faulkner, R. Jones, P. Flewitt, Radiation- and thermally-induced phosphorus inter-granular segregation in pressure vessel steels, J. ASTM International, 2 (2005) 1-15. https://doi.org/10.1520/JAI12387

[32] C. Pareige, V. Kuksenko, P. Pareige, Behaviour of P, Si, Ni impurities and Cr in self ion irradiated Fe-Cr alloys - Comparison to neutron irradiation, J. Nucl. Mater. 456 (2015) 471-476.

[33] P.D. Edmondson, C.M. Parish, R.K. Nanstad, Using complimentary microscopy methods to examine $\mathrm{Ni}-\mathrm{Mn}$-Si precipitates in highly-irradiated reactor pressure vessel steels, Acta Mater. 134 (2017) 31-39.

[34] N. Soneda, K. Dohi, A. Nomoto, K. Nishida, S. Ishino, Embrittlement correlation method for the Japanese reactor pressure vessel materials, J. ASTM International 7 (2010) 1-10. https://doi.org/10.1520/JAI102127

[35] G. Monnet, Multiscale modeling of precipitation hardening: Application to the Fe-Cr alloys, Acta Mater. 95 (2015) 302-311.

[36] G. Monnet, Multiscale modeling of irradiation hardening: Application to important nuclear materials, J. Nucl. Mater. 508 (2018) 609-627.

[37] R.S. Averback, T. Diaz de la Rubia, Displacement damage in irradiated metals and semiconductors, Solid State Physics 51 (1997) 281-402.

[38] R.E. Stoller, A.F. Calder, Statistical analysis of a library of molecular dynamics cascade simulations in iron at 100 K, J. Nucl. Mater. 283 (2000) 746-752.

[39] L. Malerba, Molecular dynamics simulation of displacement cascades in a-Fe: A critical review, J. Nucl. Mater. 351 (2006) 28-38.

[40] G.R. Odette, B.D. Wirth, A computational microscopy study of nanostructural irradiated pressure vessel steels, J. Nucl. Mater. 251 (1997) 157-171.

[41] L.C. Liu, G.R. Odette, B.D. Wirth, G.E. Lucas, A lattice Monte Carlo simulation of nanophase compositions and structures in irradiated pressure vessel $\mathrm{Fe}-\mathrm{Cu}-\mathrm{Ni}$ Mn-Si steels, Mater. Sci. Eng. A 238 (1997) 202-209.

[42] P.B. Wells, T. Yamamoto, B. Miller, T. Milot, J. Cole, Y. Wu, G.R. Odette, Evolution of manganese-nickel-silicon-dominated phases in highly irradiated reactor pressure vessel steels, Acta Mater. 80 (2014) 205-219.

[43] H. Ke, P. Wells, P.D. Edmondson, N. Almirall, L. Barnard, G.R. Odette, D. Morgan, Thermodynamic and kinetic modeling of Mn-Ni-Si precipitates in low-Cu reactor pressure vessel steels, Acta Mater. 138 (2017) 10-26.

[44] M. Mamivand, P. Wells, H. Ke, S. Shu, G.R. Odette, D. Morgan, CuMnNiSi precipitate evolution in irradiated reactor pressure vessel steels: Integrated Cluster Dynamics and experiments, Acta Mater. 180 (2019) 199-217. 
[45] N. Almirall, P.B. Wells, T. Yamamoto, K. Wilford, T. Williams, N. Riddle, G.R. Odette, Precipitation and hardening in irradiated low alloy steels with a wide range of Ni and Mn compositions, Acta Mater. 179 (2019) 119-128.

[46] R. Ngayam-Happy, C.S. Becquart, C. Domain, First principle-based AKMC modeling of the formation and medium-term evolution of point defect and solute-rich clusters in a neutron irradiated complex Fe-CuNiMnSiP alloy representative of reactor pressure vessel steels, J. Nucl. Mater. 440 (2013) 143152.

[47] R. Ngayam-Happy, C.S. Becquart, C. Domain, L. Malerba, Formation and evolution of MnNi clusters in neutron irradiated dilute Fe alloys by a first principle-based AKMC method, J. Nucl. Mater. 426 (2012) 198-207.

[48] L. Messina, M. Nastar, T. Garnier, C. Domain, P. Olsson, Exact ab initio transport coefficients in bcc Fe-X (X=Cr, Cu, Mn, Ni, P, Si) dilute alloys, Phys. Rev. B 90 (2014) 104203.

[49] L. Messina, M. Nastar, N. Sandberg, P. Olsson, Systematic electronic-structure investigation of substitutional impurity diffusion and flux coupling in bcc iron, Phys. Rev. B 93 (2016) 184302.

[50] L. Messina, Multiscale modeling of atomic transport phenomena in ferritic steels. PhD thesis (P. Olsson, N. Sandberg, \& P. Efsing, Eds.). KTH Royal Institute of Technology, Stockholm (2015).

[51] C.S. Becquart, R. Ngayam-Happy, P. Olsson, C. Domain, A DFT study of the stability of SIAs and small SIA clusters in the vicinity of solute atoms in Fe, J. Nucl. Mater. 500 (2018) 92.

[52] C. Domain, C.S. Becquart, Solute $-<111>$ interstitial loop interaction in $\alpha$-Fe: A DFT study, J. Nucl. Mater. 499 (2018) 582-594.

[53] C. Barouh, T. Schuler, C.-C. Fu, M. Nastar, Interaction between vacancies and interstitial solutes (C, N, and O) in $\alpha$-Fe: From electronic structure to thermodynamics, Phys. Rev. B 90 (2014) 054112.

[54] A. Vehanen, P. Hautojärvi, J. Johansson, J. Yli-Kauppila, P. Moser, Vacancies and carbon impurities in $\alpha$-iron: Electron irradiation, Phys. Rev. B 25 (1982) 762780.

[55] B. Minov, M. Lambrecht, D. Terentyev, C. Domain, M.J. Konstantinović, Structure of nanoscale copper precipitates in neutron-irradiated Fe-Cu-C alloys, Phys. Rev. $B 85$ (2012) 024202.

[56] D. Terentyev, A. Bakaev, E.E. Zhurkin, Effect of carbon decoration on the absorption of $<100>$ dislocation loops by dislocations in iron, J. Phys.: Condens. Matter. 26(2014) 165402.

[57] N. Anento, A. Serra, Carbon-vacancy complexes as traps for self-interstitial clusters in Fe-C alloys, J. Nucl. Mater. 440 (2013) 236-242.

[58] G. Bonny, D. Terentyev, E.E. Zhurkin, L. Malerba, Monte Carlo study of decorated dislocation loops in FeNiMnCu model alloys, J. Nucl. Mater. 452 (2014) 486-492.

[59] M.I. Pascuet, E. Martínez, G. Monnet, L. Malerba, Solute effects on edge dislocation pinning in complex alpha-Fe alloys, J. Nucl. Mater. 494 (2017) 311321.

[60] M. Chiapetto, L. Malerba, C.S. Becquart, Nanostructure evolution under irradiation in FeMnNi alloys: A "grey alloy" object kinetic Monte Carlo model, J. Nucl. Mater. 462 (2015) 91-99. 
[61] M. Chiapetto, C.S. Becquart, C. Domain, L. Malerba, Nanostructure evolution under irradiation of $\mathrm{Fe}(\mathrm{C}) \mathrm{MnNi}$ model alloys for reactor pressure vessel steels, Nucl. Instrum. Methods Phys. Res. B 352 (2015) 56-60.

[62] B.N. Singh, S.I. Golubov, H. Trinkaus, A. Serra, Yu.N. Osetsky, A.V. Barashev, Aspects of microstructure evolution and cascade damage conditions, J. Nucl. Mater. 251 (1997) 107-122.

[63] D. Terentyev, L. Malerba, M. Hou, Dimensionality of interstitial cluster motion in bcc-Fe, Phys. Rev. B 75 (2007) 104108.

[64] D. Hull, D.J. Bacon, Introduction to dislocations, Elsevier, Amsterdam, 2011, Ch. 3.

[65] L.T. Belkacemi, E. Meslin, B. Décamps, B. Radiguet, J. Henry, Radiation-induced bcc-fcc phase transformation in a Fe-3\%Ni alloy, Acta Mater. 161 (2018) 61-72.

[66] A.H. Delandar, O.I. Gorbatov, M. Selleby, Yu.N. Gornostyrev, P.A. Korzhavyi, Abinitio based search for late blooming phase compositions in iron alloys, J. Nucl. Mater. 509 (2018) 225-236.

[67] J.O. Andersson, T. Helander, L. Höglund, P.F. Shi, B. Sundman, Thermo-Calc and DICTRA, Computational tools for materials science, Calphad 26 (2002) 273-312.

[68] Y. Abe, S. Jitsukawa, Lowest energy structures of self-interstitial atom clusters in iron from a combination of Langevin molecular dynamics and the basin-hopping technique, Phil. Mag. 89 (2009) 375-388.

[69] G.R. Odette, R.K. Nanstad, Predictive reactor pressure vessel steel irradiation embrittlement models: issues and opportunities, JOM 61 (2009) 17-23.

[70] M. Burke, R. Stofanak, J. Hyde, C. English, W. Server, Microstructural aspects of irradiation damage in A508 grade $4 \mathrm{~N}$ forging steel: composition and flux effects, J. ASTM International 5 (2004) 1-14.

[71] E. Altstadt, E. Keim, H. Hein, M. Serrano, F. Bergner, H.-W. Viehrig, A. Ballesteros, R. Chaouadi, K. Wilford, FP7 Project LONGLIFE: Overview of results and implications, Nucl. Eng. Des. 278 (2014) 753-757.

[72] M. Valo, R. Krause, K. Saarinen, P. Hautojarvi, J.R. Hawthorne, Irradiation response and annealing behaviour of pressure vessel model steels and iron ternary alloys measured with positron techniques, in: R. Stoller, A. Kumar, D. Gelles (Eds.), Effects of Radiation on Materials: 15th International Symposium, ASTM International, West Coshohocken, 1992, pp. 172-185. https://doi.org/10.1520/STP17868S

[73] Y. Nagai, Z. Tang, M. Hassegawa, T. Kanai, M. Saneyasu, Irradiation-induced Cu aggregations in Fe: An origin of embrittlement of reactor pressure vessel steels, Phys. Rev. B 63 (2001) 134110.

[74] S.C. Glade, B.D. Wirth, G.R. Odette, P. Asoka-Kumar, Positron annihilation spectroscopy and small angle neutron scattering characterization of nanostructural features in high-nickel model reactor pressure vessel steels, $J$. Nucl. Mater. 351 (2006) 197-208.

[75] M.J. Konstantinović, G. Bonny, Thermal stability and the structure of vacancysolute clusters in iron alloys, Acta Mater. 85 (2015) 107-111.

[76] M.J. Konstantinovic, I. Uytdenhouwen, G. Bonny, N. Castin, L. Malerba, P. Efsing, Radiation induced solute clustering in high-Ni reactor pressure vessel steel, Acta Mater. 179 (2019) 183-189.

[77] F. Gillemot, M. Horváth, A. Horváth, B. Kovács, B. Radiguet, S. Cammelli, P. Pareige, M.H. Mayoral, A. Ulbricht, N. Kresz, F. Oszwald, G. Török, Microstructural changes in highly irradiated 15Kh2MFA steel, in: M. Kirk, E. Lucon (Eds.), Effects of Radiation on Nuclear Materials: 26th Volume, ASTM STP 1572, ASTM 
International, West Coshohocken, 2014, pp. 45-56.

https://doi.org/10.1520/STP157220130098

[78] E.A. Marquis, Atom probe tomography applied to the analysis of irradiated microstructures. J. Mater Res. 30 (2015) 1222-1230.

[79] E. Meslin, B. Radiguet, M. Loyer-Prost, Radiation-induced precipitation in a ferritic model alloy: An experimental and theoretical study, Acta Mater. 61 (2013) 6246-6254.

[80] E. Meslin, B. Radiguet, P. Pareige, C. Toffolon, A. Barbu, Irradiation-induced solute clustering in a low nickel FeMnNi ferritic alloy, Experimental Mechanics 51 (2011) 1453-1458.

[81] V. Jansson, L. Malerba, Simulation of the nanostructure evolution under irradiation in Fe-C alloys, J. Nucl. Mater. 443 (2013) 274-285.

[82] C. Domain, C.S. Becquart, Object KineticMonte Carlo (OKMC): A Coarse-Grained Approach to Radiation Damage", Springer Nature Switzerland AG 2018, W.

Andreoni, S. Yip (eds.), Handbook of Materials Modeling, https://doi.org/10.1007/978-3-319-42913-7 101-1.

[83] K.A. Fichthorn, W.H. Weinberg, Theoretical foundations of dynamical Monte Carlo simulations, J. Chem. Phys. 95 (1991) 1090-1096.

[84] M. Chiapetto, L. Malerba, C.S. Becquart, Effect of Cr content on the nanostructural evolution of irradiated ferritic/martensitic alloys: An object kinetic Monte Carlo model. J. Nucl. Mater. 465 (2015) 326-336.

[85] ASTM E693-17, Standard Practice for Characterizing Neutron Exposures in Iron and Low Alloy Steels in Terms of Displacements Per Atom, ASTM International, West Conshohocken, PA 2017. https://doi.org/10.1520/E0693-17

[86] M.J. Norgett, M.T. Robinson, I.M. Torrens, A proposed method of calculating displacement dose rates, Nucl. Eng. Des. 33 (1975) 50-54.

[87] P.J. Othen, M.L. Jenkins, G.D.W. Smith, High-resolution electron microscopy studies of the structure of $\mathrm{Cu}$ precipitates in $\alpha$-Fe, Philos. Mag. A 70 (1994) 1-24.

[88] G. Bonny, D. Terentyev, A. Bakaev, E.E. Zhurkin, M. Hou, D. Van Neck, L. Malerba, On the thermal stability of late blooming phases in reactor pressure vessel steels: An atomistic study, J. Nucl. Mater. 442 (2013) 282-291.

[89] G. Bonny, C. Domain, N. Castin, P. Olsson, L. Malerba, The impact of alloying elements on the precipitation stability and kinetics in iron based alloys: An atomistic study, Comput. Mater. Sci. 161 (2019) 309-320.

[90] J.M. Hyde, G. DaCosta, C. Hatzoglou, H. Weekes, B. Radiguet, P.D. Styman, F. Vurpillot, C. Pareige, A. Etienne, G. Bonny, N. Castin, L. Malerba, P. Pareige, Analysis of radiation damage in light water reactors: Comparison of cluster analysis methods for the analysis of atom probe data, Microsc. Microanal. 23 (2017) 366-375.

[91] L. Zhang, B. Radiguet, P. Todeschini, C. Domain, Y. Shen, P. Pareige, Investigation of solute segregation behavior using a correlative EBSD/TKD/APT methodology in a 16MND5 weld, J. Nucl. Mater. 523 (2019) 434-443

[92] S. Shu, B.D. Wirth, P.B. Wells, D.D. Morgan, G.R. Odette, Multi-technique characterization of the precipitates in thermally aged and neutron irradiated Fe$\mathrm{Cu}$ and $\mathrm{Fe}-\mathrm{Cu}-\mathrm{Mn}$ model alloys: Atom probe tomography reconstruction implications, Acta Mater. 146 (2018) 237-252.

[93] Ortner, S. et al., private communication within the LONGLIFE project (2014).

[94] N. Castin, M.I. Pascuet, L. Messina, C. Domain, P. Olsson, R.C. Pasianot, L. Malerba, Advanced atomistic models for radiation damage in Fe-based alloys: 
Contributions and future perspectives from artificial neural networks, Comput. Mater. Sci. 148 (2018) 116-130.

[95] D. Terentyev, L. Malerba, G. Bonny, A.T. Al-Motasem, M. Posselt, Interaction of an edge dislocation with Cu-Ni-Vacancy clusters in bcc iron, J. Nucl. Mater. 419 (2011) 134-139.

[96] D. Terentyev, L. Malerba, Interaction of a screw dislocation with Cu-precipitates, nanovoids and $\mathrm{Cu}$-vacancy clusters in BCC iron, Journal of Nuclear Materials 421 (2012) 32-38. 


\section{The dominating mechanisms for the formation of solute-rich clusters in steels under irradiation.}

\section{Supplementary material}

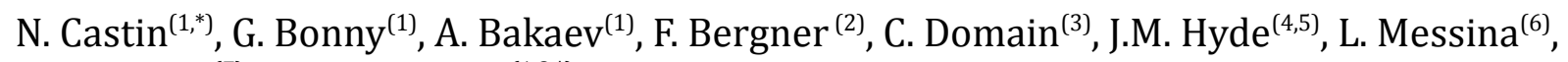
B. Radiguet ${ }^{(7)}$ and L. Malerba ${ }^{\left(1,8,{ }^{*}\right)}$

(1) Studiecentrum voor Kernenergie - Centre d'Études de l'énergie Nucléaire (SCK•CEN), NMS Unit, Boeretang 200, B2400 Mol, Belgium.

(2) Helmholtz-Zentrum Dresden-Rossendorf, Institute of Resource Ecology, P.O. Box 510119, 01314 Dresden, Germany.

(3) EDF-R\&D, Département Matériaux et Mécanique des Composants (MMC), Les Renardières, F-77818 Moret sur Loing Cedex, France.

${ }^{(4)}$ National Nuclear Laboratory, Culham Science Centre, Abingdon, Oxfordshire OX14 3DB, UK.

${ }^{(5)}$ Department of Materials, University of Oxford, Parks Road, Oxford OX1 3PH, UK

(6) KTH Royal Institute of Technology, Nuclear Engineering, 10691 Stockholm, Sweden.

(7) Groupe de Physique des Matériaux, Université et INSA de Rouen, UMR CNRS 6634, B.P. 12, 76801 Saint-Etienne du Rouvray Cedex, France.

(8) Centro de Investigaciones Energéticas, Medioambientales y Tecnológicas (CIEMAT), Avda. Complutense 40, 28040 Madrid, Spain.

${ }^{(*)}$ Corresponding author: nicolas.m.b.castin@gmail.com

\section{SM.1 Detailed parameterization of the OKMC model}

This section fully details the parameterization we adopted for the four events depicted in Fig. 5 in the main manuscript.

Except for A1, all these events correspond to the separation of a reactant in two products, denoted as $\mathrm{O} \rightarrow \mathrm{O}_{1}+\mathrm{O}_{2}$, where by convention $\mathrm{O}_{1}$ is the object that migrates away from $\mathrm{O}_{2}$. As a convention, each object $\mathrm{O}$ is described in the model by a vector $\mathrm{C}$, the components of which are its chemical composition and content in point defects, expressed by a series of integer numbers:

$$
C(O)=\left[n_{d}, n_{F e}, n_{C u}, n_{i}, n_{M n}, n_{S i}, n_{P}\right]
$$


Here, $n_{d}$ is the number of point defects in the object. By convention, if $n_{d}<0$ its module gives the number of vacancies, whereas if $n_{d}>0$ its value indicates the content in SIA. In that latter case, $n_{\mathrm{Fe}}$ indicates the content in number Fe atoms, whereas $n_{\mathrm{Cu}} n_{\mathrm{Ni}}, n_{\mathrm{Mn}}, n_{\mathrm{Si}}$ and $n_{\mathrm{P}}$ indicate the content in number of solute atoms. For convenience, we define as $n_{\text {Tot }}$ the total number of solutes:

$n_{\mathrm{Tot}}=n_{\mathrm{Cu}}+n_{\mathrm{Ni}}+n_{\mathrm{Mn}}+n_{\mathrm{Si}}+n_{\mathrm{P}}$

By assumption, only the Fe-Fe dumbbell, denoted as $[1,1,0,0,0,0,0]$, may induce an intake of Fe to a NSRC, when absorbed in a cluster.

\section{SM.1.1 Event A - Single point-defects carrying a solute atom}

These events describe the transport of solute atoms by single vacancies.

\section{Description of object 0 :}

$$
n_{d}=-1 \text { or } 1 ; n_{F e}=0 ; n_{C u}+n_{N i}+n_{M n}+n_{S i}+n_{P}=1
$$

Description of object $O_{1}$ :

$\left[n_{d}, 0, n_{\mathrm{Cu}}, n_{\mathrm{Ni}}, n_{\mathrm{Mn}}, n_{\mathrm{Si}}, n_{\mathrm{P}}\right]$ for event A1 (migration)

$\left[n_{d}, 0,0,0,0,0,0\right] \quad$ for event A2 (dissociation)

Description of object $\boldsymbol{O}_{2}$ :

$$
\begin{array}{ll}
{[0,0,0,0,0,0,0]} & \text { for event A1 (migration) } \\
{\left[0,0, n_{\mathrm{Cu}}, n_{\mathrm{Ni}}, n_{\mathrm{Mn}}, n_{\mathrm{Si}}, n_{\mathrm{P}}\right]} & \text { for event A2 (dissociation) }
\end{array}
$$

The frequencies of these events are directly calculated without approximation, in the infinite dilute limit, from migration energy data derived by L. Messina et al. [48-50]. For event A1, we write:

$$
\Gamma_{\square}^{(\text {Mig })}=\Gamma_{0}^{(\text {Mig })} \quad \exp \left(\frac{-E_{\square}^{(\text {Mig })}}{k_{B} T}\right)
$$

Where $\Gamma_{0}^{(\text {Mig) }}$ is the attempt frequency and $E_{\square}^{(\text {Mig })}$ the migration energy. Likewise, the frequency of dissociation for event A2 is described as:

$$
\Gamma_{\square}^{(\text {Diss })}=\Gamma_{0}^{(\text {Diss })} \quad \exp \left(\frac{-E_{\square}^{(\text {Diss })}}{k_{B} T}\right)
$$

Where $\Gamma_{0}^{(\text {Diss })}$ is the attempt frequency and $E_{\square}^{(\text {Diss })}$ is the dissociation energy. These parameters were evaluated performing atomistic kinetic Monte Carlo (AKMC) simulations, following a method described in Refs. [SM1]. The primary events in the AKMC model, i.e., the vacancy migration energy as a function of the solute atom coupled to it and their exact relative positions, were evaluated with DFT in Refs. [48-50]. The obtained numerical values are listed in Tab. SM1.

Table SM1 - Parameters associated with events A1 and A2 in the OKMC model, for a single vacancy coupled to a solute $S$. The case of a solute coupled with a SIA defect (forming a mixed dumbbell in the 
dilute limit) is also tabulated for $\mathrm{Mn}$ and P; other solutes do not have any binding with the SIA, therefore the event is not allowed.

\begin{tabular}{|c|c|c|c|c|}
\hline Solute $S$ & $\begin{array}{c}\Gamma_{0}^{(\text {Mig })}\left(10^{12}\right. \\
\left.s^{-1}\right)\end{array}$ & $\begin{array}{c}E_{\square}^{(\mathrm{Mig})} \\
(\mathrm{eV})\end{array}$ & $\left.\Gamma_{0}^{(\text {Diss })}{ }^{1}\right)\left(10^{12} s^{-}\right.$ & $\begin{array}{l}E_{\square}^{(\text {Diss })} \\
(\mathrm{eV})\end{array}$ \\
\hline \multicolumn{5}{|c|}{ Vacancy-S pairs } \\
\hline $\mathrm{Cu}$ & 44.3 & 0.757 & 6.190 & 0.791 \\
\hline $\mathrm{Ni}$ & 5.8 & 0.724 & 8.46 & 0.819 \\
\hline $\mathrm{Mn}$ & 44.3 & 0.757 & 6.19 & 0.791 \\
\hline $\mathrm{Si}$ & 25.4 & 0.806 & 19.2 & 0.964 \\
\hline $\mathrm{P}$ & 2.24 & 0.614 & 27.4 & 1.06 \\
\hline \multicolumn{5}{|c|}{ SIA-S mixed dumbbells } \\
\hline $\mathrm{Cu}$ & \multicolumn{4}{|c|}{ Not allowed } \\
\hline $\mathrm{Ni}$ & \multicolumn{4}{|c|}{ Not allowed } \\
\hline $\mathrm{Mn}$ & 4.22 & 0.304 & 68.5 & 0.862 \\
\hline $\mathrm{Si}$ & \multicolumn{4}{|c|}{ Not allowed } \\
\hline $\mathrm{P}$ & 2.45 & 0.141 & 25.0 & 1.07 \\
\hline
\end{tabular}

\section{SM.1.2 Event D - Single vacancy unbinds from a vacancy-solute cluster}

This event describes the interaction between a single vacancy and a vacancy-solutes cluster, determining the propensity to dissolve it or not when moving away from it.

\section{Description of object 0 :}

$$
n_{d} \leq-1 ; n_{F e} \geq 0 ; n_{C u}+n_{N i}+n_{M n}+n_{S i}+n_{P}>1
$$

\section{Description of object $O_{1}$ :}

$\left[-1,0, n_{C u}^{(e)}, n_{N i}^{(e)}, n_{M n}{ }^{(e)}, n_{S i}^{(e)}, n_{P}^{(e)}\right]$

With $n_{\text {tot }}^{(e)}=n_{C u}^{(e)}+n_{i}^{(e)}+n_{M n}^{(e)}+n_{S i}^{(e)}+n_{P}^{(e)}=1$ (vacancy-solute pair) or 0 (single vacancy)

\section{Description of object $O_{2}$ :}

$$
\left[n_{d}+1, n_{F e}, n_{C u}-n_{C u}^{(e)}, n_{N i}-n_{N i}^{(e)}, n_{M n}-n_{M n}{ }^{(e)}, n_{S i}-n_{S i}^{(e)}, n_{P}-n_{P}^{(e)}\right]
$$

Here, $n_{C u}^{(e)}, n_{N i}^{(e)}, n_{M n}^{(e)}, n_{S i}^{(e)}, n_{P}^{(e)}$ denote the solute content in the emitted object, and $n_{\text {tot }}^{(e)}$ their total number.

The migration energy $E_{O_{1}}^{(m i g)} \quad$ in Eq. 2 depends on the solute content of the migrating object $O_{1}$. If the vacancy does not carry a solute atom away from the defect ( $n_{\text {tot }}^{(e)}=0$ ), the migration energy is that for a vacancy in pure Fe, i.e. $0.63 \mathrm{eV}$. As discussed in [81], this value corresponds to the prediction provided by a (reliable) interatomic potential. The experimental value of the vacancy migration energy is 0.55 or $0.57 \mathrm{eV}$, while DFT tends to predict higher values, round $0.7 \mathrm{eV}$; the advantage of using the interatomic potential value is that: (i) it is a sort of average; (ii) more importantly, it guarantees consistency with the energy values for larger clusters, that can only be estimated using an interatomic potential. If it does carry a solute atom $\left(n_{t o t}^{(e)}=1\right)$, the corresponding value in Tab. SM1 is used.

The binding energy $E_{O_{1}, O_{2}}^{(\text {bind })}$ in Eq. 2 also depends on the solute and vacancy content of the defect. As previously mentioned, in this work we generated a table of values derived 
from DFT, covering all possible cases as long as $n_{\text {Tot }}-n_{\mathrm{d}} \leq 3$. The data are listed in section SM.3.

For the other cases $\left(n_{\text {Tot }}-n_{\mathrm{d}}>3\right)$, the estimated binding energy $\quad E_{O_{1}, O_{2}}^{(\text {bind }}\left(C\left(O_{1}\right), C\left(O_{2}\right) \|_{E s t}\right.$ in Eq. 4 should be extrapolated from the available DFT data for higher $n_{\text {Tot, }}$ up to any possible size. As explained in section 2, our understanding from the theoretical and experimental evidences about NSRC is that "nucleation" occurs heterogeneously, as a consequence of segregation of solutes on sinks for solute-dragging point-defects. Therefore, our understanding is that the free energy gain provided by the hypothetical formation of a phase can be described, in first approximation, with simplifying assumptions. In this process the nature of the solutes already segregated is assumed to play a secondary role; consistently, one can assume that the binding of a vacancy will saturate to a constant value when the size of the cluster is large enough, and will also be largely independent of the types of solute atoms located, and the presence of other vacancies, around the emitted vacancy. This saturation value needs to be estimated, assuming emission of a single vacancy or a vacancy-solute pair.

If the vacancy that is emitted drags no solute atom along with it $\left(n_{\text {tot }}^{(e)}=0\right)$, then the values in our DFT database (see section SM.3) are found to vary between $0.21 \mathrm{eV}$ and $0.83 \mathrm{eV}$, depending on the exact solutes involved. For the cases where $n_{\text {Tot }}=2$, the average value is $0.462 \mathrm{eV}$, and for the cases where $n_{\text {Tot }}=3$, the average value is $0.534 \mathrm{eV}$. Therefore, we expect the binding energy to be larger than $0.5 \mathrm{eV}$ for bigger NSRC. We thus assumed a constant value:

$$
E_{O_{1}, O_{2} \mid}^{(b i n d)}||_{\text {tost }}^{(e)}=0=i \quad 0.6 \mathrm{eV}
$$

If the vacancy that is emitted drags one solute atom along with it ( $n_{\text {tot }}^{(e)}=1$ ), then the values in our DFT database are found to vary in a larger range, between $-0.02 \mathrm{eV}$ and 1 $\mathrm{eV}$, depending on the exact composition of object 0 . For the cases where $n_{\text {Tot }}=2$, the average value is $0.172 \mathrm{eV}$, and for the cases where $n_{\text {Tot }}=3$, the average value is $0.516 \mathrm{eV}$. We again assume a constant value, to a first approximation, for all compositions of larger clusters. However, our experience with the model is that relevant predictions are obtained only if the binding energies translate the intuitive requirements that (a) small NSRC should preferably dissolve by emitting single vacancies paired with a solute atom; (b) bigger NSRC should be less likely to dissolve, and thus preferably emit vacancies not paired with solute atoms. The first feature is visible in our DFT data (small clusters), because:

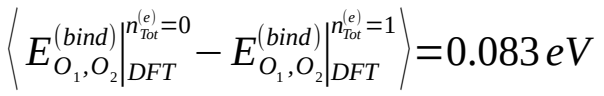

That is, the single vacancy is in average (slightly) more strongly bond to the cluster than the vacancy-solute pair. Therefore, we assume that the effective binding energy between a vacancy-solute pair and a NSRC can be approximated as follows:

$$
\begin{aligned}
\left.E_{O_{1}, O_{2}}^{(b i n)}\right|_{\text {Est }} ^{n_{\text {tot }}^{(e)}=1} & =0.52 \mathrm{eV}+\frac{\left(n_{\text {Tot }}+\left|n_{d}\right|-3\right)}{n_{\text {Sat }}-3}\left(E_{\text {Sat }}^{\text {bind }}-0.52 \mathrm{eV}\right) & & \text { if } \quad\left(n_{\text {tot }}+\left|n_{d}\right|\right)<n_{\text {Sat }} \\
& =E_{\text {Sat }}^{(b i n d)} & & \text { otherwise }
\end{aligned}
$$


With these equations, we assume that the binding energy is $0.52 \mathrm{eV}$ on average for the smaller NSRC $\left(n_{\text {Tot }}=3\right)$, whereas it linearly increases for larger sizes. The binding energy is assumed to saturate to a constant value $E_{\text {Sat }}^{(\text {bind })}$ once the NSRC is big enough, whose size is denoted as $n_{\text {Sat }}$. Our experience revealed that $n_{\text {Sat }}=65$ elements leads to satisfactory results. This number corresponds to a compact spherical cluster, surrounding the central lattice site up to the $6^{\text {th }}$ nearest neighbours distance.

\section{SM.1.3 Event B - Single SIA unbinds from a cluster of solute atoms}

This event describes the interaction between a single SIA and a solute cluster, determining the propensity to dissolve it or not while moving away from it. Note that only $\mathrm{Mn}$ and $\mathrm{P}$ atoms may be dragged away from the NSRC, because $\mathrm{Cu}, \mathrm{Ni}$ and $\mathrm{Si}$ atoms are not energetically favourable in mixed dumbbells.

\section{Description of object $O$ :}

$$
n_{d}=1 ; n_{F e} \geq 0 ; n_{C u}+n_{N i}+n_{M n}+n_{S i}+n_{P}>1
$$

\section{Description of object $O_{1}$ :}

$$
\left[1,0,0,0, n_{M n}{ }^{(e)}, 0, n_{P}{ }^{(e)}\right]
$$

With $n_{\text {tot }}^{(e)}=n_{M n}^{(e)}+n_{P}^{(e)}=1$ (mixed dumbbell emitted) or 0 (Fe-Fe dumbbell emitted)

\section{Description of object $\mathrm{O}_{2}$ :}

$$
\left[0, n_{F e}, n_{C u}, n_{N i}, n_{M n}-n_{M n}{ }^{(e)}, n_{S i}, n_{P}-n_{P}{ }^{(e)}\right]
$$

The migration energy $E_{O_{1}}^{(m i g)} \quad$ in Eq. 2 depends on the solute content of the migrating object $O_{1}$. If the SIA does not carry a solute atom away from the solute cluster $\left(n_{\text {tot }}^{(e)}=0\right.$ ), the migration energy is the one of the SIA in pure Fe, i.e. $0.31 \mathrm{eV}$ [SM2]. If it does carry a solute atom ( $n_{\text {tot }}^{(e)}=1$ ), we take the corresponding value in Table SM1.

The binding energy $E_{O_{1}, O_{2}}^{(b i n d)}$ in Eq. 2 also depends on the solute content of the migrating object. It should thus, ideally, be evaluated with DFT, e.g. up to $n_{\text {Tot }}+n_{\mathrm{d}}=3$ as for the single vacancy case (see event E in section SM.1.2). Considering that this event is expected to be relatively rare, however, we considered it sufficient, to a first approximation, to roughly estimate the binding energy as follows:

If the SIA drags no solute atom along with it ( $n_{\text {tot }}^{(e)}=0$ ), we assume that the effective binding energy is governed by the highest binding between a single SIA and any of the solutes found in the NSRC. Therefore:

$$
E_{O_{1}, O_{2}}^{(b i n)} \mid \begin{aligned}
& n_{\text {tost }}^{(e)}=0 \\
& \text { (e) }
\end{aligned}=\max _{S}\left(E_{\text {SIA }, S}^{(\text {bind })} \cdot\left(1-\delta_{n_{S}}\right)\right)
$$

Here, $\mathrm{S}$ denotes the chemical species ( $\mathrm{S}=\mathrm{Cu}, \mathrm{Ni}, \mathrm{Mn}, \mathrm{Si}$ or P), and $n_{\mathrm{S}}$ the number of corresponding solutes in the object 0 , and $\delta_{n_{s}}$ is Kronecker's symbol ( $\delta_{n_{S}}=1$ if $n_{S}$ ¿0 while $\delta_{n_{S}}=0$ if $n_{\mathrm{S}} \quad \dot{0} \quad$ ). Finally, $\quad E_{\text {SIA,S }, \text { (bind }}$ is the binding energy between a single SIA and a single solute atom S. The values estimated with DFT are provided in Tab. SM2 
Table SM2 - Binding energies in eV, evaluated with DFT, between a single SIA and a solute S (first line), and between a $37\langle 111\rangle$ SIA loop and a solute S (second line)

\begin{tabular}{|l|l|l|l|l|l|l|}
\hline & $\mathrm{Cu}$ & $\mathrm{Ni}$ & $\mathrm{Mn}$ & $\mathrm{Si}$ & $\mathrm{P}$ & Ref \\
\hline$E_{\text {SIA, } S}^{(\text {bind }}$ & $0.13 \mathrm{eV}$ & $0.15 \mathrm{eV}$ & $0.39 \mathrm{eV}$ & $0.19 \mathrm{eV}$ & $1.02 \mathrm{eV}$ & {$[\mathrm{SM} 3]$} \\
\hline$E_{\text {Loop }, S}^{(\text {bind }}$ & $0.40 \mathrm{eV}$ & $0.20 \mathrm{eV}$ & $0.37 \mathrm{eV}$ & $0.40 \mathrm{eV}$ & $1.06 \mathrm{eV}$ & {$[52]$} \\
\hline
\end{tabular}

If the SIA drags one solute atom along with it $\left(n_{\text {tot }}^{(e)}=1\right)$, a similar reasoning as the discussion above in section SM.1.2 can be followed. For simplicity, in first approximation, we followed the same logic, and therefore applied Eq. SM7 and Eq. SM8 using the same parameters.

\section{SM.1.4 Event C - SIA loop unbinds from a cluster of solute atoms}

This event describes how the mobility of loops is affected by decorating solutes.

\section{Description of object $O$ :}

$$
n_{d}>1 ; n_{F e} \geq 0 ; n_{C u}+n_{N i}+n_{M n}+n_{S i}+n_{P}>0
$$

\section{Description of object $O_{1}$ :}

$$
\left[n_{d}, 0,0,0,0,0\right]
$$

\section{Description of object $O_{2}$ :}

$$
\left[0, n_{\mathrm{Fe}}, n_{\mathrm{Cu}}, n_{\mathrm{Ni}}, n_{\mathrm{Mn}}, n_{\mathrm{Si}}, n_{\mathrm{P}}\right]
$$

The emission of a single SIA from an SIA cluster is already highly unlikely in pure Fe [68], thus it is neglected here as well. Since the loop cannot transport solutes on long distances while migrating [64], the only possibility left is a detachment of the loop from the whole cluster of solute atoms, that will obviously become the less likely, the more solutes decorate the loop. DFT data concerning the binding between SIA loops and individual solute atoms are provided in [52], for up to two solutes decorating the loop. The DFT data for two solutes are shown in that work to be roughly additive. We thus assume that, as long as the number of solutes is small, they will interact very little with each other, especially if located close to the edge of the loop where the interaction is the strongest. Hence, in order to estimate $E_{O_{1}, O_{2}}^{\text {bind }}$ in Eq. 2, we assume, to a first approximation, that decorating solutes have independent and attractive binding actions on the loop, for any number of solutes:

$$
\left.E_{O_{1}, O_{2} \mid}^{(b i n d)}\right|_{E s t} ^{P}=\sum_{S=C u, \ni, M n, S i, P}^{\unrhd} E_{S, n_{I}}^{(\text {bind })} \cdot n_{S}
$$

Here, $E_{S, n_{I}}^{(b i n d)}$ is the maximum value (edge) provided in [52], as summarized in Tab. SM2. 
The figures below provide extra information concerning the composition of the NSRC as predicted by our OKMC model. Like in the manuscript, different colours denote different values for $E_{\text {Sat }}^{(b i n d)}$ in Eq. SM7.

On the left panels, the Fe content in the NSRC according to the model is reported. As explained in the main document, this balance accounts for the number of SIA defects, free of solute atoms (no mixed dumbbells), that were absorbed by the solute clusters. This is therefore our estimation, based on the assumption that Fe can only be brought to NSRC in this way, irrespectively of the hypothesized artefacts inherent to the APT. We can see that the predicted Fe content is quite low, in the majority of cases lower than $10 \%$. It is also found to increase with decreasing $E_{\text {Sat }}^{\text {bind }}$. This can be explained in the following way. Decreasing $E_{\text {Sat }}^{\text {bind }}$ favours the release of single vacancies from solute clusters. Indirectly, this extra content of single vacancies in the material bulk contributes to eliminating single SIA defects. Their reduced content in the materials thus reduces the amount of Fe atoms brought to the NSRC.

On the right panels, the proportion of vacancies decorated by solutes is reported. Here, vacancies are counted as single defects. For example, a di-vacancy cluster is counted twice as a single vacancy. We count the number of single vacancies, irrespective of how many there are in the cluster, $N_{v}{ }^{c l}$, that belong to a complex that contains more than 5 solutes $\left(N_{s}^{c l}\right.$ is the total number of solutes in the cluster, $\left.N_{s}^{c l}>5\right)$. The curves correspond to the ratio $N_{v}{ }^{c l} / N_{s}^{c l}$. We see that this ratio varies with the value of $E_{\text {Sat }}^{(b i n)}$, as well. It increases with $E_{\text {Sat }}^{(b i n)}$, as a direct consequence of increased binding between solutes and vacancies. In any case, we conclude that the majority of the vacancies are likely to be found in defects (single vacancies or vacancy clusters) decorated with little amounts of solute atoms.

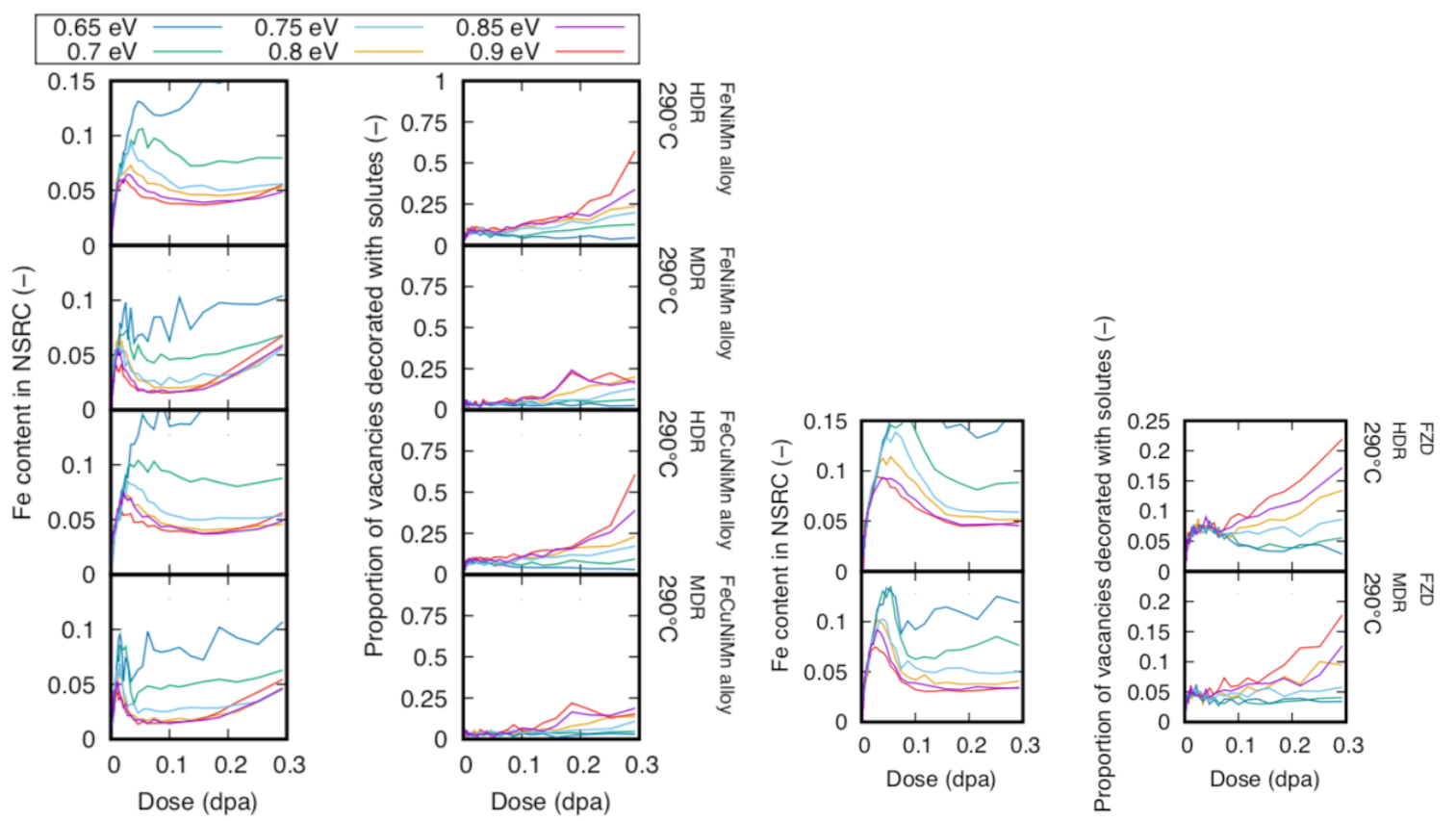



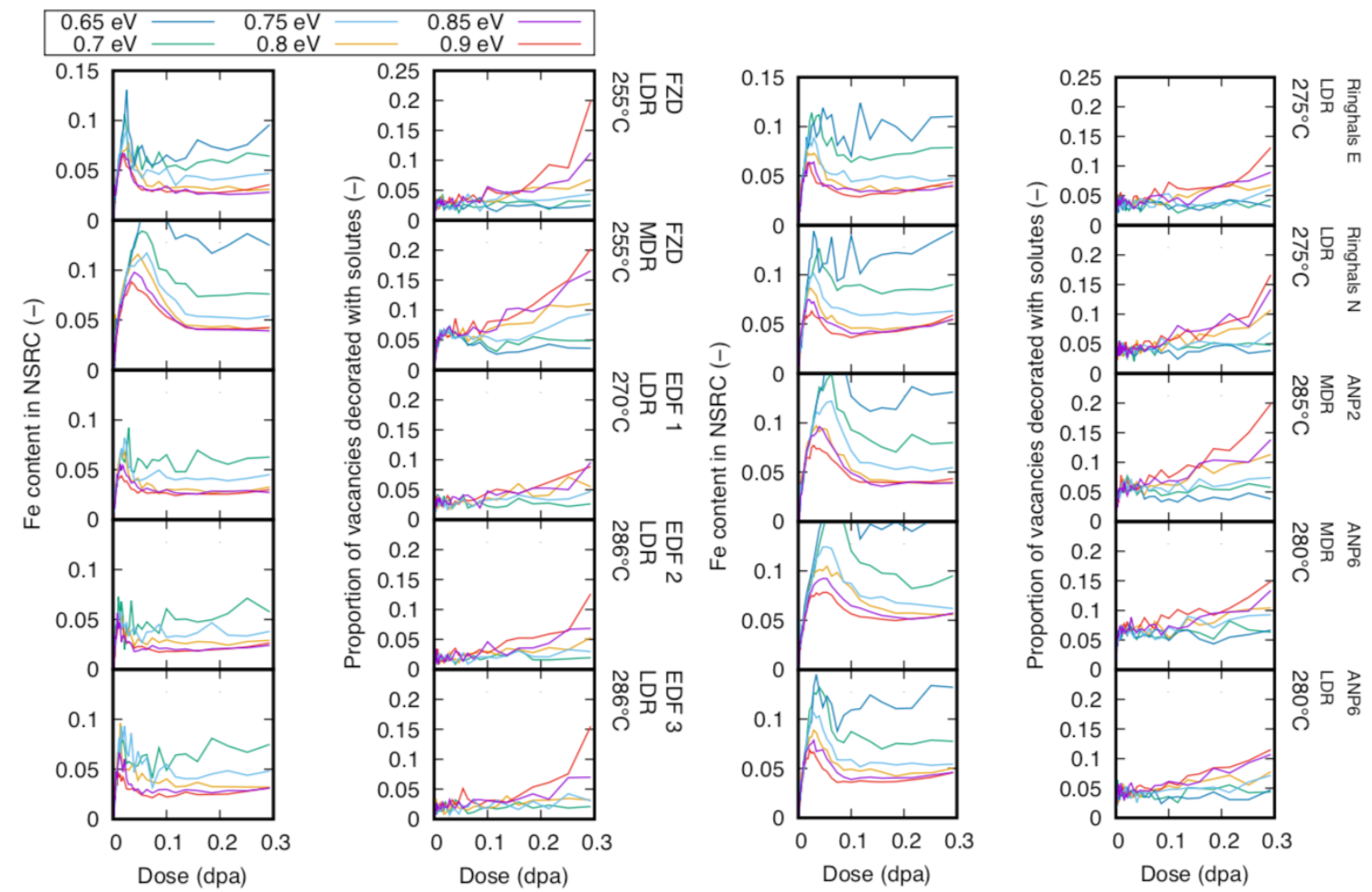

\section{SM.3 Parameters obtained with density functional theory}

In Eq. 3, the application of the OKMC model requires the knowledge of the value of the binding energy between a vacancy (optionally coupled to a solute atom) and a small cluster of solute atoms. This energy was evaluated by DFT calculations for up to quadruplets of solute atoms, using VASP [SM4, SM5]. The calculations have been performed using pseudopotentials from the VASP library, which were generated within the projector augmented wave (PAW) approach [SM6]. They were spin polarized and the exchange-correlation functional was described by the generalized gradient approximation (GGA) of Perdew et al. [SM7], with the Vosko-Wilk-Nusair (VWN) interpolation of the correlation energy [SM8]. Periodic boundary conditions and the supercell approach have been used for all calculations with 128 atoms and a lattice parameter of $0.2831 \mathrm{~nm}$. The Brillouin zone has been sampled using the MonkhorstPack scheme [SM9], with $3 \times 3 \times 3 k$-points. The calculation of defect energies have been performed at constant volume for all the configurations, thus relaxing only the atomic positions.

The binding energies calculated with DFT are listed in the following tables. The first six columns indicate the chemical composition of the solute cluster in the initial state 0 . The next six columns indicate the chemical composition of object $\mathrm{O}_{1}$, i.e., what solute atom is dragged along with the vacancy, while moving away from the cluster. Finally, the last column gives the binding energy estimated with DFT. Positive values mean attractive interactions, and vice versa. 


\begin{tabular}{|c|c|c|c|c|c|c|c|c|c|c|c|c|}
\hline \multicolumn{6}{|c|}{ Object 0 composition } & \multicolumn{6}{|c|}{ Object $\mathrm{O}_{1}$ composition } & \multirow{2}{*}{$E_{O_{1}, O_{2}}^{\mid \text {bind }}(e l$} \\
\hline$n_{V d}$ & $n_{i}$ & $n$ & $n_{S i}^{\square}$ & $n$ & $n$ & $n_{V a}$ & $n_{i}^{(e)}$ & $n_{M}^{(e)}$ & $n_{S i}^{(e)}$ & $n_{P}^{(e}$ & $n_{C u}^{(e)}$ & \\
\hline \multirow[t]{2}{*}{1} & \multirow[t]{2}{*}{0} & \multirow[t]{2}{*}{0} & \multirow[t]{2}{*}{0} & \multirow[t]{2}{*}{0} & \multirow[t]{2}{*}{2} & 1 & 0 & 0 & 0 & 0 & 0 & 0.49 \\
\hline & & & & & & 1 & 0 & 0 & 0 & 0 & 1 & 0.27 \\
\hline \multirow[t]{2}{*}{1} & \multirow[t]{2}{*}{0} & \multirow[t]{2}{*}{0} & \multirow[t]{2}{*}{0} & \multirow[t]{2}{*}{0} & \multirow[t]{2}{*}{3} & 1 & 0 & 0 & 0 & 0 & 0 & 0.56 \\
\hline & & & & & & 1 & 0 & 0 & 0 & 0 & 1 & 1.01 \\
\hline \multirow[t]{3}{*}{1} & \multirow[t]{3}{*}{0} & \multirow[t]{3}{*}{0} & \multirow[t]{3}{*}{0} & \multirow[t]{3}{*}{1} & 1 & 1 & 0 & 0 & 0 & 0 & 0 & 0.54 \\
\hline & & & & & & 1 & 0 & 0 & 0 & 1 & 0 & 0.22 \\
\hline & & & & & & 1 & 0 & 0 & 0 & 0 & 1 & 0.34 \\
\hline 1 & 0 & 0 & 0 & 1 & 2 & 1 & 0 & 0 & 0 & 0 & 0 & 0.55 \\
\hline & & & & & & 1 & 0 & 0 & 0 & 1 & 0 & 0.89 \\
\hline & & & & & & 1 & 0 & 0 & 0 & 0 & 1 & 0.82 \\
\hline 1 & 0 & 0 & 0 & 2 & 0 & 1 & 0 & 0 & 0 & 0 & 0 & 0.75 \\
\hline & & & & & & 1 & 0 & 0 & 0 & 1 & 0 & 0.31 \\
\hline 1 & 0 & 0 & 0 & 2 & 1 & 1 & 0 & 0 & 0 & 0 & 0 & 0.75 \\
\hline & & & & & & 1 & 0 & 0 & 0 & 1 & 0 & 0.66 \\
\hline & & & & & & 1 & 0 & 0 & 0 & 0 & 1 & 0.97 \\
\hline 1 & 0 & 0 & 0 & 3 & 0 & 1 & 0 & 0 & 0 & 0 & 0 & 0.83 \\
\hline & & & & & & 1 & 0 & 0 & 0 & 1 & 0 & 0.66 \\
\hline 1 & 0 & 0 & 1 & 0 & 1 & 1 & 0 & 0 & 0 & 0 & 0 & 0.47 \\
\hline & & & & & & 1 & 0 & 0 & 1 & 0 & 0 & 0.16 \\
\hline & & & & & & 1 & 0 & 0 & 0 & 0 & 1 & 0.2 \\
\hline 1 & 0 & 0 & 1 & 0 & 2 & 1 & 0 & 0 & 0 & 0 & 0 & 0.5 \\
\hline & & & & & & 1 & 0 & 0 & 1 & 0 & 0 & 0.75 \\
\hline & & & & & & 1 & 0 & 0 & 0 & 0 & 1 & 0.69 \\
\hline 1 & 0 & 0 & 1 & 1 & 0 & 1 & 0 & 0 & 0 & 0 & 0 & 0.61 \\
\hline & & & & & & 1 & 0 & 0 & 1 & 0 & 0 & 0.19 \\
\hline & & & & & & 1 & 0 & 0 & 0 & 1 & 0 & 0.11 \\
\hline 1 & 0 & 0 & 1 & 1 & 1 & 1 & 0 & 0 & 0 & 0 & 0 & 0.61 \\
\hline & & & & & & 1 & 0 & 0 & 1 & 0 & 0 & 0.35 \\
\hline & & & & & & 1 & 0 & 0 & 0 & 1 & 0 & 0.48 \\
\hline & & & & & & 1 & 0 & 0 & 0 & 0 & 1 & 0.8 \\
\hline 1 & 0 & 0 & 1 & 2 & 0 & 1 & 0 & 0 & 0 & 0 & 0 & 0.74 \\
\hline & & & & & & 1 & 0 & 0 & 1 & 0 & 0 & 0.56 \\
\hline & & & & & & 1 & 0 & 0 & 0 & 1 & 0 & 0.44 \\
\hline 1 & 0 & 0 & 2 & 0 & 0 & 1 & 0 & 0 & 0 & 0 & 0 & 0.51 \\
\hline & & & & & & 1 & 0 & 0 & 1 & 0 & 0 & 0.05 \\
\hline 1 & 0 & 0 & 2 & 0 & 1 & 1 & 0 & 0 & 0 & 0 & 0 & 0.57 \\
\hline & & & & & & 1 & 0 & 0 & 1 & 0 & 0 & 0.32 \\
\hline & & & & & & 1 & 0 & 0 & 0 & 0 & 1 & 0.65 \\
\hline 1 & 0 & 0 & 2 & 1 & 0 & 1 & 0 & 0 & 0 & 0 & 0 & 0.62 \\
\hline & & & & & & 1 & 0 & 0 & 1 & 0 & 0 & 0.39 \\
\hline & & & & & & 1 & 0 & 0 & 0 & 1 & 0 & 0.21 \\
\hline 1 & 0 & 0 & 3 & 0 & 0 & 1 & 0 & 0 & 0 & 0 & 0 & 0.54 \\
\hline & & & & & & 1 & 0 & 0 & 1 & 0 & 0 & 0.02 \\
\hline
\end{tabular}




\begin{tabular}{|c|c|c|c|c|c|c|c|c|c|c|c|c|}
\hline \multirow[t]{3}{*}{1} & \multirow[t]{3}{*}{0} & \multirow[t]{3}{*}{1} & \multirow[t]{3}{*}{0} & \multirow[t]{3}{*}{0} & \multirow[t]{3}{*}{1} & 1 & 0 & 0 & 0 & 0 & 0 & 0.49 \\
\hline & & & & & & 1 & 0 & 1 & 0 & 0 & 0 & 0.25 \\
\hline & & & & & & 1 & 0 & 0 & 0 & 0 & 1 & 0.16 \\
\hline \multirow[t]{3}{*}{1} & \multirow[t]{3}{*}{0} & \multirow[t]{3}{*}{1} & \multirow[t]{3}{*}{0} & \multirow[t]{3}{*}{0} & \multirow[t]{3}{*}{2} & 1 & 0 & 0 & 0 & 0 & 0 & 0.67 \\
\hline & & & & & & 1 & 0 & 1 & 0 & 0 & 0 & 0.57 \\
\hline & & & & & & 1 & 0 & 0 & 0 & 0 & 1 & 0.88 \\
\hline \multirow[t]{3}{*}{1} & \multirow[t]{3}{*}{0} & \multirow[t]{3}{*}{1} & \multirow[t]{3}{*}{0} & \multirow[t]{3}{*}{1} & \multirow[t]{3}{*}{0} & 1 & 0 & 0 & 0 & 0 & 0 & 0.5 \\
\hline & & & & & & 1 & 0 & 1 & 0 & 0 & 0 & 0.29 \\
\hline & & & & & & 1 & 0 & 0 & 0 & 1 & 0 & 0.08 \\
\hline \multirow[t]{4}{*}{1} & \multirow[t]{4}{*}{0} & 1 & 0 & 1 & 1 & 1 & 0 & 0 & 0 & 0 & 0 & 0.8 \\
\hline & & & & & & 1 & 0 & 1 & 0 & 0 & 0 & 0.72 \\
\hline & & & & & & 1 & 0 & 0 & 0 & 1 & 0 & 0.9 \\
\hline & & & & & & 1 & 0 & 0 & 0 & 0 & 1 & 0.68 \\
\hline 1 & 0 & 1 & 0 & 2 & 0 & 1 & 0 & 0 & 0 & 0 & 0 & 0.54 \\
\hline & & & & & & 1 & 0 & 1 & 0 & 0 & 0 & 0.54 \\
\hline & & & & & & 1 & 0 & 0 & 0 & 1 & 0 & 0.33 \\
\hline 1 & 0 & 1 & 1 & 0 & 0 & 1 & 0 & 0 & 0 & 0 & 0 & 0.38 \\
\hline & & & & & & 1 & 0 & 1 & 0 & 0 & 0 & 0.17 \\
\hline & & & & & & 1 & 0 & 0 & 1 & 0 & 0 & 0.04 \\
\hline 1 & 0 & 1 & 1 & 0 & 1 & 1 & 0 & 0 & 0 & 0 & 0 & 0.4 \\
\hline & & & & & & 1 & 0 & 1 & 0 & 0 & 0 & 0.33 \\
\hline & & & & & & 1 & 0 & 0 & 1 & 0 & 0 & 0.51 \\
\hline & & & & & & 1 & 0 & 0 & 0 & 0 & 1 & 0.36 \\
\hline 1 & 0 & 1 & 1 & 1 & 0 & 1 & 0 & 0 & 0 & 0 & 0 & 0.53 \\
\hline & & & & & & 1 & 0 & 1 & 0 & 0 & 0 & 0.49 \\
\hline & & & & & & 1 & 0 & 0 & 1 & 0 & 0 & 0.01 \\
\hline & & & & & & 1 & 0 & 0 & 0 & 1 & 0 & 0.2 \\
\hline 1 & 0 & 1 & 2 & 0 & 0 & 1 & 0 & 0 & 0 & 0 & 0 & 0.45 \\
\hline & & & & & & 1 & 0 & 1 & 0 & 0 & 0 & 0.43 \\
\hline & & & & & & 1 & 0 & 0 & 1 & 0 & 0 & 0.13 \\
\hline 1 & 0 & 2 & 0 & 0 & 0 & 1 & 0 & 0 & 0 & 0 & 0 & 0.31 \\
\hline & & & & & & 1 & 0 & 1 & 0 & 0 & 0 & 0.11 \\
\hline 1 & 0 & 2 & 0 & 0 & 1 & 1 & 0 & 0 & 0 & 0 & 0 & 0.29 \\
\hline & & & & & & 1 & 0 & 1 & 0 & 0 & 0 & 0.16 \\
\hline & & & & & & 1 & 0 & 0 & 0 & 0 & 1 & 0.32 \\
\hline 1 & 0 & 2 & 0 & 1 & 0 & 1 & 0 & 0 & 0 & 0 & 0 & 0.61 \\
\hline & & & & & & 1 & 0 & 1 & 0 & 0 & 0 & 0.63 \\
\hline & & & & & & 1 & 0 & 0 & 0 & 1 & 0 & 0.82 \\
\hline 1 & 0 & 2 & 1 & 0 & 0 & 1 & 0 & 0 & 0 & 0 & 0 & 0.22 \\
\hline & & & & & & 1 & 0 & 1 & 0 & 0 & 0 & 0.27 \\
\hline & & & & & & 1 & 0 & 0 & 1 & 0 & 0 & 0.4 \\
\hline 1 & 0 & 3 & 0 & 0 & 0 & 1 & 0 & 0 & 0 & 0 & 0 & 0.22 \\
\hline & & & & & & 1 & 0 & 1 & 0 & 0 & 0 & 0.34 \\
\hline 1 & 1 & 0 & 0 & 0 & 1 & 1 & 0 & 0 & 0 & 0 & 0 & 0.39 \\
\hline & & & & & & 1 & 1 & 0 & 0 & 0 & 0 & 0.3 \\
\hline & & & & & & 1 & 0 & 0 & 0 & 0 & 1 & 0.15 \\
\hline
\end{tabular}




\begin{tabular}{|c|c|c|c|c|c|c|c|c|c|c|c|c|}
\hline \multirow[t]{3}{*}{1} & \multirow[t]{3}{*}{1} & \multirow[t]{3}{*}{0} & \multirow[t]{3}{*}{0} & \multirow[t]{3}{*}{0} & \multirow[t]{3}{*}{2} & 1 & 0 & 0 & 0 & 0 & 0 & 0.44 \\
\hline & & & & & & 1 & 1 & 0 & 0 & 0 & 0 & 0.59 \\
\hline & & & & & & 1 & 0 & 0 & 0 & 0 & 1 & 0.8 \\
\hline \multirow[t]{3}{*}{1} & \multirow[t]{3}{*}{1} & \multirow[t]{3}{*}{0} & \multirow[t]{3}{*}{0} & \multirow[t]{3}{*}{1} & \multirow[t]{3}{*}{0} & 1 & 0 & 0 & 0 & 0 & 0 & 0.45 \\
\hline & & & & & & 1 & 1 & 0 & 0 & 0 & 0 & 0.28 \\
\hline & & & & & & 1 & 0 & 0 & 0 & 1 & 0 & 0.01 \\
\hline \multirow[t]{4}{*}{1} & \multirow[t]{4}{*}{1} & \multirow[t]{4}{*}{0} & \multirow[t]{4}{*}{0} & \multirow[t]{4}{*}{1} & \multirow[t]{4}{*}{1} & 1 & 0 & 0 & 0 & 0 & 0 & 0.61 \\
\hline & & & & & & 1 & 1 & 0 & 0 & 0 & 0 & 0.7 \\
\hline & & & & & & 1 & 0 & 0 & 0 & 1 & 0 & 0.79 \\
\hline & & & & & & 1 & 0 & 0 & 0 & 0 & 1 & 0.72 \\
\hline \multirow[t]{3}{*}{1} & \multirow[t]{3}{*}{1} & 0 & 0 & 2 & 0 & 1 & 0 & 0 & 0 & 0 & 0 & 0.64 \\
\hline & & & & & & 1 & 1 & 0 & 0 & 0 & 0 & 0.72 \\
\hline & & & & & & 1 & 0 & 0 & 0 & 1 & 0 & 0.54 \\
\hline 1 & 1 & 0 & 1 & 0 & 0 & 1 & 0 & 0 & 0 & 0 & 0 & 0.39 \\
\hline & & & & & & 1 & 1 & 0 & 0 & 0 & 0 & 0.17 \\
\hline & & & & & & 1 & 0 & 0 & 1 & 0 & 0 & -0.02 \\
\hline 1 & 1 & 0 & 1 & 0 & 1 & 1 & 0 & 0 & 0 & 0 & 0 & 0.54 \\
\hline & & & & & & 1 & 1 & 0 & 0 & 0 & 0 & 0.58 \\
\hline & & & & & & 1 & 0 & 0 & 1 & 0 & 0 & 0.66 \\
\hline & & & & & & 1 & 0 & 0 & 0 & 0 & 1 & 0.58 \\
\hline 1 & 1 & 0 & 1 & 1 & 0 & 1 & 0 & 0 & 0 & 0 & 0 & 0.5 \\
\hline & & & & & & 1 & 1 & 0 & 0 & 0 & 0 & 0.59 \\
\hline & & & & & & 1 & 0 & 0 & 1 & 0 & 0 & 0.16 \\
\hline & & & & & & 1 & 0 & 0 & 0 & 1 & 0 & 0.37 \\
\hline 1 & 1 & 0 & 2 & 0 & 0 & 1 & 0 & 0 & 0 & 0 & 0 & 0.47 \\
\hline & & & & & & 1 & 1 & 0 & 0 & 0 & 0 & 0.47 \\
\hline & & & & & & 1 & 0 & 0 & 1 & 0 & 0 & 0.24 \\
\hline 1 & 1 & 1 & 0 & 0 & 0 & 1 & 0 & 0 & 0 & 0 & 0 & 0.37 \\
\hline & & & & & & 1 & 1 & 0 & 0 & 0 & 0 & 0.17 \\
\hline & & & & & & 1 & 0 & 1 & 0 & 0 & 0 & 0.12 \\
\hline 1 & 1 & 1 & 0 & 0 & 1 & 1 & 0 & 0 & 0 & 0 & 0 & 0.56 \\
\hline & & & & & & 1 & 1 & 0 & 0 & 0 & 0 & 0.46 \\
\hline & & & & & & 1 & 0 & 1 & 0 & 0 & 0 & 0.44 \\
\hline & & & & & & 1 & 0 & 0 & 0 & 0 & 1 & 0.67 \\
\hline 1 & 1 & 1 & 0 & 1 & 0 & 1 & 0 & 0 & 0 & 0 & 0 & 0.7 \\
\hline & & & & & & 1 & 1 & 0 & 0 & 0 & 0 & 0.59 \\
\hline & & & & & & 1 & 0 & 1 & 0 & 0 & 0 & 0.64 \\
\hline & & & & & & 1 & 0 & 0 & 0 & 1 & 0 & 0.83 \\
\hline 1 & 1 & 1 & 1 & 0 & 0 & 1 & 0 & 0 & 0 & 0 & 0 & 0.21 \\
\hline & & & & & & 1 & 1 & 0 & 0 & 0 & 0 & 0.18 \\
\hline & & & & & & 1 & 0 & 1 & 0 & 0 & 0 & 0.16 \\
\hline & & & & & & 1 & 0 & 0 & 1 & 0 & 0 & 0.35 \\
\hline 1 & 1 & 2 & 0 & 0 & 0 & 1 & 0 & 0 & 0 & 0 & 0 & 0.36 \\
\hline & & & & & & 1 & 1 & 0 & 0 & 0 & 0 & 0.34 \\
\hline & & & & & & 1 & 0 & 1 & 0 & 0 & 0 & 0.33 \\
\hline 1 & 2 & 0 & 0 & 0 & 0 & 1 & 0 & 0 & 0 & 0 & 0 & 0.28 \\
\hline
\end{tabular}




\begin{tabular}{|c|c|c|c|c|c|c|c|c|c|c|c|c|}
\hline & & & & & & 1 & 1 & 0 & 0 & 0 & 0 & 0.17 \\
\hline \multirow[t]{3}{*}{1} & \multirow[t]{3}{*}{2} & \multirow[t]{3}{*}{0} & \multirow[t]{3}{*}{0} & \multirow[t]{3}{*}{0} & \multirow[t]{3}{*}{1} & 1 & 0 & 0 & 0 & 0 & 0 & 0.43 \\
\hline & & & & & & 1 & 1 & 0 & 0 & 0 & 0 & 0.44 \\
\hline & & & & & & 1 & 0 & 0 & 0 & 0 & 1 & 0.59 \\
\hline \multirow[t]{3}{*}{1} & \multirow[t]{3}{*}{2} & \multirow[t]{3}{*}{0} & \multirow[t]{3}{*}{0} & \multirow[t]{3}{*}{1} & \multirow[t]{3}{*}{0} & 1 & 0 & 0 & 0 & 0 & 0 & 0.62 \\
\hline & & & & & & 1 & 1 & 0 & 0 & 0 & 0 & 0.63 \\
\hline & & & & & & 1 & 0 & 0 & 0 & 1 & 0 & 0.73 \\
\hline \multirow[t]{3}{*}{1} & \multirow[t]{3}{*}{2} & \multirow[t]{3}{*}{0} & \multirow[t]{3}{*}{1} & \multirow[t]{3}{*}{0} & \multirow[t]{3}{*}{0} & 1 & 0 & 0 & 0 & 0 & 0 & 0.53 \\
\hline & & & & & & 1 & 1 & 0 & 0 & 0 & 0 & 0.49 \\
\hline & & & & & & 1 & 0 & 0 & 1 & 0 & 0 & 0.6 \\
\hline \multirow[t]{3}{*}{1} & \multirow[t]{3}{*}{2} & \multirow[t]{3}{*}{1} & \multirow[t]{3}{*}{0} & \multirow[t]{3}{*}{0} & \multirow[t]{3}{*}{0} & 1 & 0 & 0 & 0 & 0 & 0 & 0.54 \\
\hline & & & & & & 1 & 1 & 0 & 0 & 0 & 0 & 0.51 \\
\hline & & & & & & 1 & 0 & 1 & 0 & 0 & 0 & 0.41 \\
\hline \multirow[t]{2}{*}{1} & 3 & 0 & 0 & 0 & 0 & 1 & 0 & 0 & 0 & 0 & 0 & 0.38 \\
\hline & & & & & & 1 & 1 & 0 & 0 & 0 & 0 & 0.41 \\
\hline 2 & 1 & 0 & 0 & 0 & 0 & 1 & 0 & 0 & 0 & 0 & 0 & 0.23 \\
\hline & & & & & & 1 & 1 & 0 & 0 & 0 & 0 & 0.23 \\
\hline 2 & 0 & 1 & 0 & 0 & 0 & 1 & 0 & 0 & 0 & 0 & 0 & 0.24 \\
\hline & & & & & & 1 & 0 & 1 & 0 & 0 & 0 & 0.24 \\
\hline 2 & 0 & 0 & 1 & 0 & 0 & 1 & 0 & 0 & 0 & 0 & 0 & 0.4 \\
\hline & & & & & & 1 & 0 & 0 & 1 & 0 & 0 & 0.4 \\
\hline 2 & 0 & 0 & 0 & 1 & 0 & 1 & 0 & 0 & 0 & 0 & 0 & 0.56 \\
\hline & & & & & & 1 & 0 & 0 & 0 & 1 & 0 & 0.56 \\
\hline 2 & 0 & 0 & 0 & 0 & 1 & 1 & 0 & 0 & 0 & 0 & 0 & 0.32 \\
\hline & & & & & & 1 & 0 & 0 & 0 & 0 & 1 & 0.32 \\
\hline 3 & 1 & 0 & 0 & 0 & 0 & 1 & 0 & 0 & 0 & 0 & 0 & 0.7 \\
\hline & & & & & & 1 & 1 & 0 & 0 & 0 & 0 & 0.8 \\
\hline 3 & 0 & 1 & 0 & 0 & 0 & 1 & 0 & 0 & 0 & 0 & 0 & 0.76 \\
\hline & & & & & & 1 & 0 & 1 & 0 & 0 & 0 & 0.82 \\
\hline 3 & 0 & 0 & 1 & 0 & 0 & 1 & 0 & 0 & 0 & 0 & 0 & 0.81 \\
\hline & & & & & & 1 & 0 & 0 & 1 & 0 & 0 & 1 \\
\hline 3 & 0 & 0 & 0 & 1 & 0 & 1 & 0 & 0 & 0 & 0 & 0 & 1.1 \\
\hline & & & & & & 1 & 0 & 0 & 0 & 1 & 0 & 1.33 \\
\hline 3 & 0 & 0 & 0 & 0 & 1 & 1 & 0 & 0 & 0 & 0 & 0 & 1.05 \\
\hline & & & & & & 1 & 0 & 0 & 0 & 0 & 1 & 0.93 \\
\hline
\end{tabular}

References

[1-96] See main manuscript.

[SM1] Pascuet, M.I., Castin, N., Becquart, C.S., Malerba, L. Stability and mobility of Cuvacancy clusters in $\mathrm{Fe}-\mathrm{Cu}$ alloys: A computational study based on the use of 
artificial neural networks for energy barrier calculations. J. Nucl. Mater. 412, 106-115 (2011).

[SM2] M.-C. Marinica, F. Willaime, N. Mousseau, Energy landscape of small clusters of self-interstitial dumbbells in iron, Physical Review B 83 (2011) 094119.

[SM3] Ngayam-Happy, R., Becquart, C.S., Domain, C. First principle-based AKMC modelling of the formation and medium-term evolution of point defect and solute-rich clusters in a neutron irradiated complex Fe-CuMnNiSiP alloy representative of reactor pressure vessel steels. J. Nucl. Mater. 440, 143-152 (2013).

[SM4] Kresse, G. \& Hafner, J. Ab initio molecular dynamics for liquid metals. Phys. Rev. B. 47, 558 (1993).

[SM5] Kresse, G. \& Furthmüller, J. Efficient iterative schemes for $a b$ initio total-energy calculations using a plane-wave basis set. Phys. Rev. B 54, 11169 (1996).

[SM6] Kresse, G. \& Joubert, D. From ultrasoft pseudopotentials to the projector augmented-wave method. Phys. Rev. B 59, 1758 (1999).

[SM7] Perdew, J. P., et al. Atoms, molecules, solids, and surfaces: Applications of the generalized gradient approximation for exchange and correlation. Phys. Rev. B 4, 6671 (1992).

[SM8] Wilk, L., Nusair, M. \& Vosko, S. H. The Fermi contact contribution to the Knight shift in Be from self-consistent spin-polarized calculations. Can. J. Phys. 59 585595 (1981).

[SM9] Monkhorst, H. J. \& Pack, J. D. Special points for Brillouin-zone integrations. Phys. Rev. B 13, 5188 (1976). 